\title{
Marine boundary layer cloud property retrievals from high-resolution ASTER observations: case studies and comparison with Terra MODIS
}

\author{
Frank Werner ${ }^{1}$, Galina Wind ${ }^{2}$, Zhibo Zhang ${ }^{3}$, Steven Platnick ${ }^{2}$, Larry Di Girolamo ${ }^{4}$, Guangyu Zhao $^{4}$, \\ Nandana Amarasinghe ${ }^{5}$, and Kerry Meyer $^{2,6}$ \\ ${ }^{1}$ Joint Center for Earth Systems Technology, 5523 Research Park Drive, Baltimore, \\ MD 21228, USA \\ ${ }^{2}$ NASA Goddard Space Flight Center, Greenbelt, MD, 20771, USA \\ ${ }^{3}$ Physics Department, University of Maryland, Baltimore County, 1000 Hilltop Circle, \\ Baltimore, MD 21228, USA \\ ${ }^{4}$ Department of Atmospheric Sciences, University of Illinois at Urbana-Champaign, \\ 105 South Gregory Street, Urbana, IL 61801, USA \\ ${ }^{5}$ Science Systems and Applications, Inc., NASA/GSFC, Greenbelt, MD 20771, USA \\ ${ }^{6}$ Goddard Earth Sciences Technology and Research (GESTAR), Universities Space Research \\ Association, Columbia, MD 21046, USA
}

Correspondence to: Frank Werner (frankw@umbc.edu)

Received: 5 August 2016 - Published in Atmos. Meas. Tech. Discuss.: 9 August 2016

Revised: 1 November 2016 - Accepted: 17 November 2016 - Published: 8 December 2016

\begin{abstract}
A research-level retrieval algorithm for cloud optical and microphysical properties is developed for the Advanced Spaceborne Thermal Emission and Reflection Radiometer (ASTER) aboard the Terra satellite. It is based on the operational MODIS algorithm. This paper documents the technical details of this algorithm and evaluates the retrievals for selected marine boundary layer cloud scenes through comparisons with the operational MODIS Data Collection 6 (C6) cloud product. The newly developed, ASTERspecific cloud masking algorithm is evaluated through comparison with an independent algorithm reported in Zhao and Di Girolamo (2006). To validate and evaluate the cloud optical thickness $(\tau)$ and cloud effective radius $\left(r_{\text {eff }}\right)$ from ASTER, the high-spatial-resolution ASTER observations are first aggregated to the same $1000 \mathrm{~m}$ resolution as MODIS. Subsequently, $\tau_{\mathrm{aA}}$ and $r_{\mathrm{eff}}$ aA retrieved from the aggregated ASTER radiances are compared with the collocated MODIS retrievals. For overcast pixels, the two data sets agree very well with Pearson's product-moment correlation coefficients of $R>0.970$. However, for partially cloudy pixels there are significant differences between $r_{\text {eff, aA }}$ and the MODIS re-
\end{abstract}

sults which can exceed $10 \mu \mathrm{m}$. Moreover, it is shown that the numerous delicate cloud structures in the example marine boundary layer scenes, resolved by the high-resolution ASTER retrievals, are smoothed by the MODIS observations. The overall good agreement between the research-level ASTER results and the operational MODIS C6 products proves the feasibility of MODIS-like retrievals from ASTER reflectance measurements and provides the basis for future studies concerning the scale dependency of satellite observations and three-dimensional radiative effects.

\section{Introduction}

The shortwave radiative effects of marine boundary layer (MBL) clouds on the climate system are largely determined by cloud fraction, cloud optical thickness $\tau$ and effective

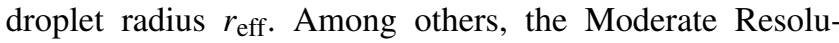
tion Imaging Spectroradiometer (MODIS) provides global retrievals of these cloud variables. However, derived cloud properties from passive remote sensing techniques are in- 
herently dependent on the spatial resolution of the observations and thus the influence of cloud horizontal heterogeneities. Studies on scale-dependent uncertainties in estimated cloud amount due to unresolved clear-sky contaminations have been reported by Shenk and Salomonson (1972), Wielicki and Parker (1992), DiGirolamo and Davies (1997), and Dey et al. (2008). This dependence on spatial resolution also extents to the retrievals of $\tau$ and $r_{\text {eff }}$, which usually are achieved with the help of the bispectral solar reflective method (Twomey and Seton, 1980; Nakajima and King, 1990; Rossow and Schiffer, 1991) and under the independent pixel approximation (IPA; see Cahalan et al., 1994a, b).

The IPA introduces two general three-dimensional (3-D) radiative effects, caused by applying one-dimensional (1-D) radiative transfer on 3-D cloud structures. For observations with a high spatial resolution, such as the Thematic Mapper onboard the Landsat satellites, cloud heterogeneities at scales larger than the sensor spatial resolution yield a breakdown of IPA (Barker and Liu, 1995; Chambers et al., 1997). In contrast, satellite observations with a lower spatial resolution cannot resolve heterogeneous cloud structures within a pixel, introducing significant biases in retrieved $\tau$ and $r_{\text {eff }}$ (Cahalan et al., 1994a; Marshak et al., 2006; Zhang and Platnick, 2011; Zhang et al., 2012). Depending on the spatial resolution of the observations, the biases due to resolved or unresolved variability can vary in magnitude and even compensate each other to a certain degree (Marshak et al., 2006). The increase in sensor spatial resolution of spaceborne instruments, while introducing increased detail in retrieved cloud parameters even for heterogeneous cloud fields, compounds the importance of 3-D radiative transfer in the cloudy atmosphere. The bispectral retrieval approach is adopted by several major satellite missions, most prominently by the MODIS instrument. MODIS provides operational cloud products sampled on a global scale with a horizontal resolution of $1000 \mathrm{~m}$, which makes the retrieved MODIS cloud variables susceptible to biases introduced by resolved and unresolved variability.

Studies on satellite observations of unresolved cloud variability require a significantly higher spatial resolution. Samples by the Advanced Spaceborne Thermal Emission and Reflection Radiometer (ASTER) are characterized by a horizontal resolution as high as $15 \mathrm{~m}$ (Abrams, 2000). While ASTER data are usually applied to study changes in land cover and biophysical parameters (Stefanov and Netzband, 2005), there are a number of studies deploying ASTER for cloud observations. Zhao and Di Girolamo (2006, 2007) and Dey et al. (2008) use the high-resolution ASTER reflectance measurements at the $\lambda=0.86 \mu \mathrm{m}$ band to derive a statistical description of the macrophysical properties of trade wind clouds. Hulley and Hook (2008) describe a number of spectral tests to distinguish cloudy observations from those over different surfaces and cloud shadows. Seiz et al. (2006) and Genkova et al. (2007) employ the stereoscopic capabilities of ASTER, as well as its window infrared (IR) window-channel, for studying cloud top heights. Jones et al. (2012) use the high-resolution ASTER data as a training set for a pattern recognition approach for a new algorithm implemented by the MISR team to improve cloud fraction estimates. Finally, Marshak et al. (2006) and Wen et al. (2007) report on MODIS cloud property retrievals and apply ASTER reflectances to gain a better understanding of the cloud 3-D structure on the MODIS microphysical cloud retrievals. Despite all these studies, there remains no retrieval algorithm that provides cloud top, optical, and microphysical properties for the high-resolution ASTER observations.

In this study MODIS-like retrievals of $\tau$ and $r_{\text {eff }}$ based on high-resolution ASTER observations are presented. Both ASTER and MODIS are aboard NASA's Terra satellite, which allows for intercomparison studies and cross validation of the retrieval products. The objectives of this study are as follows: (i) documenting the research-level retrieval setup, which provides cloud property retrievals based on ASTER observations, and (ii) comparing co-located ASTER retrievals with the operational MODIS C6 results for 48 selected MBL scenes with different degrees of horizontal heterogeneity. It is shown that estimates of $\tau$ and $r_{\text {eff }}$ from ASTER measurements are consistent with the operational MODIS data products. Thus, the combination of highresolution ASTER observations and the presented retrieval setup provides a unique framework for future studies on the reliability of retrievals for partially cloudy pixels, the scale dependence of satellite-based remote sensing products and the influence of 3-D radiative effects.

The paper is structured as follows: an overview of ASTER and MODIS, as well as the difference between important spectral bands of the two instruments, is given in Sect. 2. The applied cloud masking scheme and the ASTER-specific cloud property retrieval algorithm are presented in Sect. 3 . Subsequently, a comparison of the retrieval products between the operational MODIS C6 and collocated ASTER results is shown in Sect. 5, followed by a summary in Sect. 6 .

\section{ASTER and MODIS}

This section provides an introduction to the ASTER instrument and a detailed description of the steps necessary to obtain reflectances from the raw ASTER observations. A brief overview of MODIS is also given. Differences between the spectral response functions (SRFs) of both instruments are presented.

\subsection{ASTER}

ASTER is an imaging spectroradiometer installed aboard the multinational scientific research satellite Terra (EOS AM-1). Information on the instrument design and science objectives can be found in Yamaguchi and Hiroji (1993), Yamaguchi et al. (1998), and Abrams (2000). ASTER collects data in 15 
Table 1. Overview of ASTER band numbers, wavelength range $(\Delta \lambda)$ covered by each band, spatial resolution $(\Delta x)$, as well as unit conversion coefficients $\left(C_{\mathrm{H}}, C_{\mathrm{N}}, C_{\mathrm{L} 1}, C_{\mathrm{L} 2}\right)$ for high, normal, low-1 and low-2 gains, respectively.

\begin{tabular}{|c|c|c|c|c|c|c|}
\hline Band & $\begin{array}{r}\Delta \lambda \\
(\mu \mathrm{m})\end{array}$ & $\begin{array}{l}\Delta x \\
(\mathrm{~m})\end{array}$ & $\mathrm{W} \mathrm{m}^{-2} \mu^{-1} \mathrm{C}_{\mathrm{H}}$ & $\mathrm{W} \mathrm{m}^{-2} \mu_{\mathrm{m}^{-1}}^{C_{\mathrm{N}}} \mathrm{sr}$ & $\mathrm{W} \mathrm{m}^{-2} \mu_{\mathrm{m}}^{-1}{ }_{\mathrm{sr}}$ & 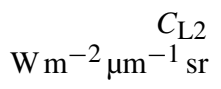 \\
\hline 1 & $0.520-0.600$ & 15 & 0.676 & 1.688 & 2.25 & - \\
\hline 2 & $0.630-0.690$ & 15 & 0.708 & 1.415 & 1.89 & - \\
\hline $3 \mathrm{~N}$ & $0.760-0.860$ & 15 & 0.423 & 0.862 & 1.15 & - \\
\hline $3 \mathrm{~B}$ & $0.760-0.860$ & 15 & 0.423 & 0.862 & 1.15 & - \\
\hline 4 & $1.600-1.700$ & 30 & 0.1087 & 0.2174 & 0.290 & 0.290 \\
\hline 5 & $2.145-2.185$ & 30 & 0.0348 & 0.0696 & 0.0925 & 0.409 \\
\hline 6 & $2.185-2.225$ & 30 & 0.0313 & 0.0625 & 0.0830 & 0.390 \\
\hline 7 & $2.235-2.285$ & 30 & 0.0299 & 0.0597 & 0.0795 & 0.332 \\
\hline 8 & $2.295-2.365$ & 30 & 0.0209 & 0.0417 & 0.0556 & 0.245 \\
\hline 9 & $2.360-2.430$ & 30 & 0.0159 & 0.0318 & 0.0424 & 0.265 \\
\hline 10 & $8.125-8.475$ & 90 & - & 0.006822 & - & - \\
\hline 11 & $8.475-8.825$ & 90 & - & 0.006780 & - & - \\
\hline 12 & $8.925-9.275$ & 90 & - & 0.006590 & - & - \\
\hline 13 & $10.250-10.950$ & 90 & - & 0.005693 & - & - \\
\hline 14 & $10.950-11.650$ & 90 & - & 0.005225 & - & - \\
\hline
\end{tabular}

distinct spectral bands, covering the visible to the thermal IR spectral wavelength range. The spatial resolution of an individual ASTER pixel in the visible to near-infrared spectral wavelength range (VNIR) is $15 \mathrm{~m}$, while it is 30 and $90 \mathrm{~m}$ in the in the shortwave-infrared (SWIR) and IR spectral wavelength range, respectively. Table 1 lists the spectral band numbers and the respective wavelength ranges $\Delta \lambda$ that the ASTER instrument covers. While all bands are operated in nadir-viewing mode, the $\lambda=0.760-0.860 \mu \mathrm{m}$ band also provides a backward-viewing direction. From the 10:30 (local time) sun-synchronous orbit of Terra, ASTER samples roughly 650 scenes daily. Each of these scenes covers an area of $60 \times 60 \mathrm{~km}^{2}$. However, ASTER data sampled over ocean surfaces are usually not archived and observations of MBL clouds are provided by specific scientific objectives, as reviewed in Jones et al. (2012).

The digital ASTER counts $d_{\mathrm{A}}(\Delta \lambda)$ that are sampled by the instrument over a cloud scene are converted into spectral ASTER radiances $I_{\mathrm{A}}(\Delta \lambda)$ via the conversion equation provided by Abrams et al. (2004):

$I_{\mathrm{A}}(\Delta \lambda)=\left(d_{\mathrm{A}}(\Delta \lambda)-1\right) \cdot C_{\mathrm{N}}$.

The unit conversion coefficient $C_{\mathrm{N}}$ for each band, which is dependent on the respective gain setting provided in the embedded metadata of each ASTER data container, is also given in Table 1 . Spectral ASTER reflectances $R_{\mathrm{A}}\left(\Delta \lambda, \theta_{0}\right)$ are calculated by

$R_{\mathrm{A}}\left(\Delta \lambda, \theta_{0}\right)=\frac{\pi \cdot I_{\mathrm{A}}(\Delta \lambda) \cdot r_{\mathrm{SE}}^{2}}{F_{0}(\Delta \lambda) \cdot \cos \theta_{0}}$

where $r_{\mathrm{SE}}$ denotes the distance between the Earth and the Sun in astronomical units and $F_{0}(\Delta \lambda)$ is the incoming spectral solar irradiance modified by the solar zenith angle $\theta_{0}$.
Band-specific $F_{0}(\Delta \lambda)$ values are obtained from the convolution of high-resolution spectral solar irradiances with the respective SRF of each ASTER band. The high-resolution spectral solar irradiance of $\lambda=0.199-0.539 \mu \mathrm{m}$ is provided by Thuillier et al. (2003), $\lambda=0.540-1.060 \mu \mathrm{m}$ by Neckel and Labs (1984), and $\lambda=1.450-400.000 \mu \mathrm{m}$ by Thekaekara (1974). The specific ASTER response functions are provided by the ASTER science team on the instrument website (http://asterweb.jpl.nasa.gov/characteristics.asp).

Absolute radiometric uncertainties $\delta$ of VNIR and SWIR reflectances are $<4 \%$ (Yamaguchi et al., 1998). However, due to an increase in the SWIR detector temperature starting in May 2007, the SWIR signal started to suffer from anomalous striping and saturation of values. While the VNIR and IR bands are not affected, no reliable SWIR data sampled after that date are available (with brief exceptions in June and July 2007, as well as January to April 2008).

\subsection{MODIS}

The scanning radiometer MODIS is installed aboard NASA's Terra and Aqua (EOS PM-1) platforms, launched in 1999 and 2002, respectively. MODIS has a viewing swath width of $2330 \mathrm{~km}$. Together with the orbit characteristics of the Terra (and Aqua) platform this allows for a global coverage every 2 days. MODIS collects data in 36 spectral bands between 0.415 and $14.235 \mu \mathrm{m}$. Except for a number of bands, the general spatial resolution of a MODIS pixel is $1000 \mathrm{~m}$. Further information on MODIS and the cloud product algorithms is given in Ardanuy et al. (1992), Barnes et al. (1998), and Platnick et al. (2003).

The current version of the cloud product algorithm, and the one that yields the data in this study, is Data Collection 6 

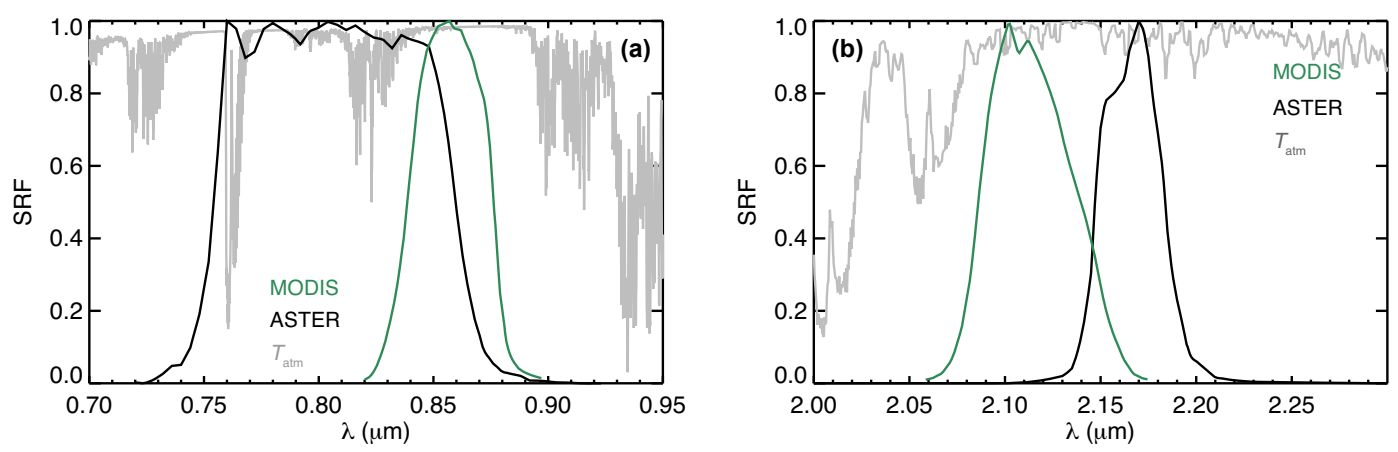

Figure 1. (a) Spectral response function (SRF) of the VNIR band signal for MODIS (green) and ASTER (black) as a function of wavelength ( $\lambda$ ). Gray lines indicate the atmospheric transmittance $T_{\text {atm }}$, calculated for the US 1976 Standard Atmosphere assuming a nadir-viewing geometry and a solar zenith angle of $\theta_{0}=0^{\circ}$. (b) Same as (a) but for the signal in the SWIR band.

(C6). This new set of algorithms includes a flag for partially cloudy (PCL) pixels.

\subsection{Comparison of spectral response functions}

The MODIS cloud property retrieval is based on reflectances sampled in two spectral bands, one in the VNIR and one in the SWIR. Although ASTER employs similar bands in these spectral regions, differences in the respective SRF can impact the retrieval. It is therefore important to understand the behavior of the ASTER SRFs and the respective band deviations from the MODIS instrument. For MODIS the VNIR reflectance $R_{0.86, \mathrm{M}}$ is provided by band 2 , which covers $\lambda=0.841-0.876 \mu \mathrm{m}$ and is centered around $\lambda=0.8585 \mu \mathrm{m}$, while the SWIR reflectances $R_{2.1, \mathrm{M}}$ are sampled by band 7 , which covers $\lambda=2.105-2.155 \mu \mathrm{m}$ and is centered around $\lambda=2.130 \mu \mathrm{m}$. VNIR and SWIR reflectances $R_{0.86, \mathrm{~A}}$ and $R_{2.1, \mathrm{~A}}$ for ASTER are detected at bands $3 \mathrm{~N}$ (nadir-viewing mode) and 5 , respectively.

Figure 1a shows the SRF of the ASTER (black) and MODIS (green) VNIR band as a function of wavelength $\lambda$. Compared to MODIS, the SRF of the ASTER VNIR band is significantly broader with a spectral width of about $\Delta \lambda=$ $0.100 \mu \mathrm{m}$ (compared to $\Delta \lambda=0.060 \mu \mathrm{m}$ for MODIS). Moreover, the center of the SRF is shifted by about $\lambda=0.050 \mu \mathrm{m}$ towards smaller wavelengths. The center position and width of the ASTER VNIR band implies that measurements are affected by important absorption features of atmospheric oxygen $\left(\mathrm{O}_{2}\right.$ A-band around $\left.0.760 \mu \mathrm{m}\right)$ and water vapor (mainly between 0.810 and $0.840 \mu \mathrm{m}$ ). These features become apparent in the atmospheric transmittance spectrum $T_{\mathrm{atm}}$ (gray), which was derived by simulations with the MODerate resolution atmospheric TRANsmission (MODTRAN) code version 4.2r1 (Berk et al., 1998), assuming profiles for atmospheric gases following the US 1976 Standard Atmosphere (NASA, 1976). The atmospheric correction scheme, which accounts for these absorption features, as well as associated uncertainties is described in Sects. 3.2 and 5.4.
Figure 1b shows the SRF of the applied ASTER and MODIS SWIR bands, respectively. Compared to MODIS, the center of the ASTER SRF is shifted by about $\lambda=$ $0.035 \mu \mathrm{m}$ towards larger wavelengths and the spectral width is decreased by about $\Delta \lambda=0.004 \mu \mathrm{m}$.

Implications of the SRF differences on the cloud property retrieval are discussed in Sect. 3.3.

\section{Cloud property retrieval algorithm}

In this section an ASTER-specific cloud masking scheme is presented in detail. The derived scene cloud covers and pixel-level statistics for 124 ASTER cases are compared to those calculated from single-band thresholds developed on a scene-by-scene basis. Subsequently the research-level ASTER cloud property retrieval algorithm is documented.

\subsection{Cloud detection for ASTER}

Cloud detection from moderate to high-resolution imagers can take on many forms, from simple single thresholding approaches to more elaborate machine learning approaches. As clearly demonstrated in Yang and Di Girolamo (2008), cloud detection algorithms must be designed with a particular purpose in mind. The retrieval algorithm presented in Sect. 3.2 is a research-level algorithm and is specifically employed to study the effects of sensor resolution on remote sensing products of MBL clouds. For this reason, the highest resolution available from ASTER $(15 \mathrm{~m})$ is targeted, while the need for an operationally complete and globally validated cloud detection algorithm is not required at this time. Still, the manually tedious effort to produce scene-by-scene cloud masks for a multitude of different resolutions based on a single (or more) threshold approach (e.g., Wielicki and Welch, 1986, Zhao and Di Girolamo, 2006) is replaced in favor of a hybrid approach. Here, individual cases are selected based on the presence of low-level water clouds over the ocean and the absence of high-level cirrus that impacts the cloud 
property retrieval (Wind et al., 2010). Subsequently, a simple decision-tree approach (e.g., Saunders and Kriebel, 1988; Ackerman et al., 1998) was developed, using five thresholding tests to produce a $15 \mathrm{~m}$ resolution cloud mask. Since the focus of this study is on the feasibility of cloud microphysical retrievals from ASTER, the cloud masking scheme is cloud-conservative. Following the MODIS cloud mask designation presented in Platnick et al. (2003), ASTER pixels can be flagged as confidently cloudy, probably cloudy, probably clear, or confidently clear. The five cloudiness tests performed are described below:

i. ASTER band $3 \mathrm{~N}$ reflectances $R_{0.86, \mathrm{~A}}$ need to exceed distinct thresholds. Similar tests to identify clear-sky pixels have been reported by Ackerman et al. (1998), Ackerman et al. (2008), Frey et al. (2008), and Banks and Mélin (2015) for MODIS observations, generally establishing thresholds of $R_{0.86, \mathrm{M}}<0.03$ for confidently clear and $R_{0.86, \mathrm{M}}>0.065$ for cloudy pixels.

ii. Similar to test (i), a threshold for ASTER band 5 reflectances $R_{2.1 \text {, A }}$ is defined to distinguish between clouds and the darker ocean surface.

iii. A ratio of ASTER band $3 \mathrm{~N}$ and band 2 reflectances, calculated as $r_{1}=\frac{R_{0.86, A}}{R_{0.65, A}}$, is applied to distinguish clouds from the darker ocean surface, as well as from measurement over land. This ratio utilizes the rather constant spectral behavior of clouds in the VNIR, which leads to their white appearance. Ackerman et al. (1998) found thresholds of $0.8<r_{1}<1.1$, while Ackerman et al. (2008) and Banks and Mélin (2015) reported adjusted lower thresholds of 0.85 and 0.95 for confidently clear and cloudy pixels, respectively. The upper threshold is usually set to 1.1, in part to exclude land surfaces. Tests with different ASTER cases have shown that this value can reach values of $r_{1}>1.3$ for cloud observations, while land surfaces show $r_{1} \gg 1.3$. Tests (i)-(iii) are usually sufficient for identifying reasonably bright cumulus clouds (i.e., $R_{0.86, \mathrm{~A}} \geq 0.2$ ).

iv. To better distinguish cloud edges and very thin cumuli from the ocean surface it proves helpful to define a second ratio in the VNIR. The ratio of ASTER band 1 and band 2 reflectances, calculated as $r_{2}=\frac{R_{0.52, A}}{R_{0.65, A}}$, shows rather large values of $r_{2}>1.6$ over the ocean due to increased Rayleigh scattering (i.e., the VNIR spectrum in this range has a steeper slope). Similar to $r_{1}$ this ratio is close to 1 for cloudy pixels, because of their spectrally invariant behavior in the VNIR.

Categorizing pixels into confidently cloudy, probably cloudy, probably clear, and confidently clear pixels is performed with the decision tree illustrated in Fig. 3. The derived thresholds for tests (i)-(iv) are as follows. Confidently cloudy pixels (cloudiness flag “0”) indicate pixels with sufficiently large
ASTER band $3 \mathrm{~N}$ reflectances and either contain bright low level cumuli or clouds with a large vertical extent. These pixels are identified by $R_{0.86, A}>0.065, R_{2.1, A}>0.02,0.80<$ $r_{1}<1.75$, and $r_{2}<1.2$. Probably cloudy pixels (cloudiness flag " 1 ") are associated with observations covering rather thin clouds and cloud edges. They are characterized by lower band $3 \mathrm{~N}$ reflectances. These pixels are identified by $R_{0.86, A}>0.03, R_{2.1, A}>0.015,0.75<r_{1}<1.75$, and $r_{2}<$ 1.35. Probably clear pixels (cloudiness flag " 2 ") are characterized by $R_{0.86, A}>0.03, R_{2.1, A}>0.01,0.70<r_{1}<1.75$, and $r_{2}<1.45$. Usually, these pixels are clear. However, if such pixels are flagged as cloudy, a cloud property retrieval either fails or yields an ASTER cloud optical thickness $\tau_{\mathrm{A}}<$ 5. All other pixels are identified as clear (cloudiness flag "3"). These thresholds, which comprise the first step in the new cloud masking scheme, were set through inspection of 210 ASTER MBL scenes sampled off the coast of California and the tropical western Atlantic (Zhao and Di Girolamo, 2006) between April 2003 and July 2007. These observations have been performed at full ASTER resolution, keeping in mind that we are siding on a cloud-conservative cloud mask. While the thresholds are derived for a wide range of solar zenith angles (observed $\theta_{0}=33.4-63.2^{\circ}$ for the $210 \mathrm{MBL}$ scenes), aerosol optical depths (observed 0.04-1.49 for the $210 \mathrm{MBL}$ scenes), and even a small number of sunglint cases, they are static with no dependence on $\theta_{0}$. As demonstrated below, the quality of the cloud mask tests meets the purpose of this study and is believed to be more broadly appropriate for deep ocean scenes, in atmospheres with low aerosol turbidity, and outside of strong sunglint and large $\theta_{0}$. However, it should be noted that further refinements of these thresholds are likely for investigations outside the scope of this study.

Due to increased horizontal photon transport in more complex broken cumulus scenes (where there is a large number of cumuli with small horizontal extent), as well as cases with pronounced sunglint, it is found that tests (i)-(iv) can become noisy and sometimes falsely identify clear pixels as cloudy. Therefore, in a second step a threshold for the brightness temperature $T_{\mathrm{B}, 11}$, derived from the ASTER Band 14 radiances, is defined to correctly label such pixels as clear-sky observations. This threshold $\left(T_{\mathrm{B}, \mathrm{c} 5}\right.$, cloudiness test $\left.\mathrm{v}\right)$ is calculated as the 5 th percentile of $T_{\mathrm{B}, 11}$ sampled over all clear pixels (cloudiness flag " 3 ") if the fraction of clear pixels $n_{\mathrm{c}}$ in the respective scene is at least 0.03 . This guarantees a sufficient number of samples to calculate frequency distributions of $T_{\mathrm{B}}$ (e.g., even for a horizontal resolution of $1000 \mathrm{~m}$ over 100 clear pixels remain). In order to match the spatial resolution of the VNIR observations, each $T_{\mathrm{B}, 11}$ sample at $90 \mathrm{~m}$ resolution is replicated onto 36 subpixels with a horizontal resolution of $15 \mathrm{~m}$.

Figure $2 \mathrm{a}-\mathrm{e}$ show the results of the five thresholding tests for a broken cumulus case observed over the tropical western Atlantic on 2 December 2004 at 14:17 UTC. This scene is characterized by a multitude of individual cumuli with small horizontal extent and a low scene cloud cover of $C_{\mathrm{A}}=0.04$. 

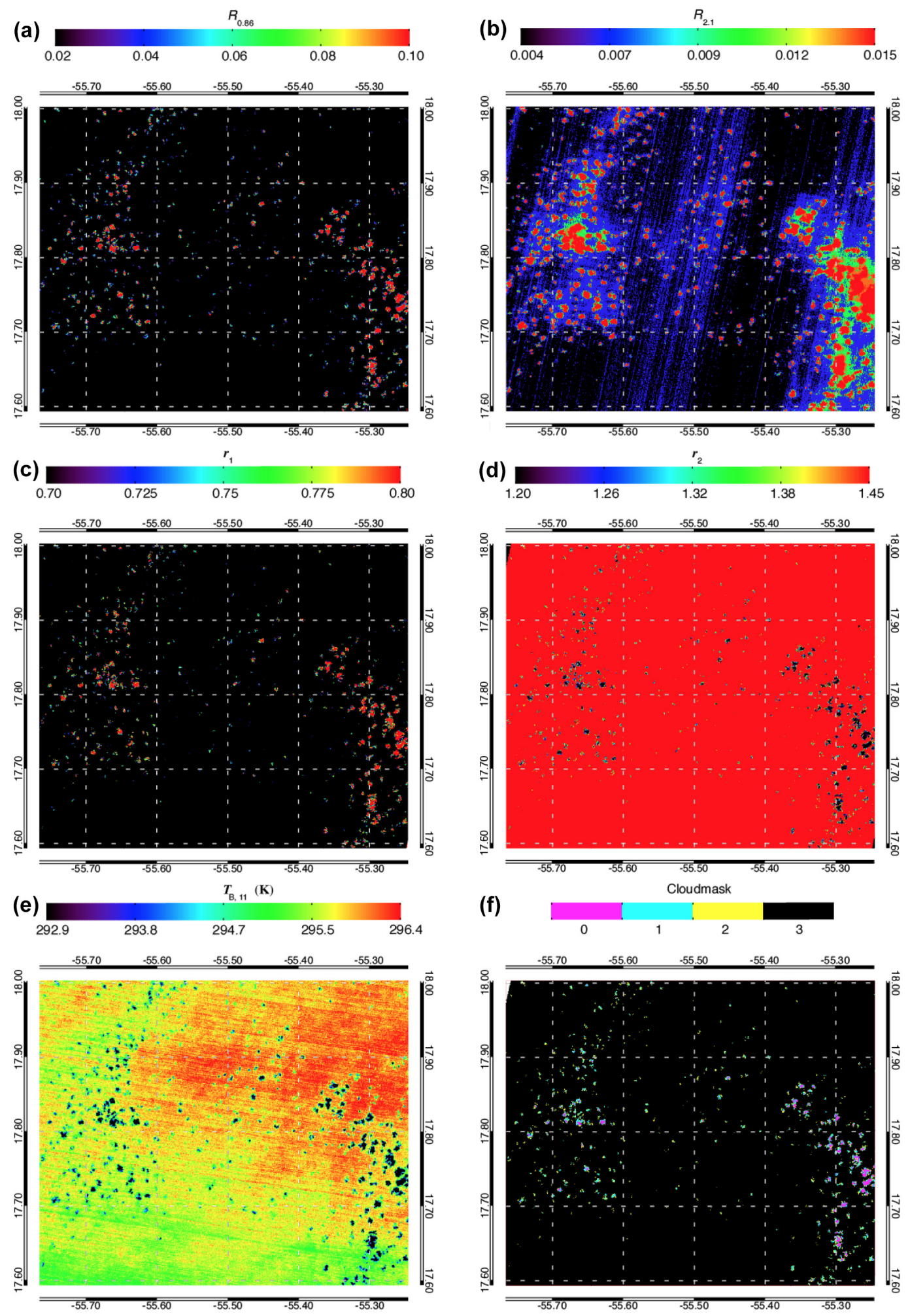

Figure 2. (a) Band $3 \mathrm{~N}$ reflectance $\left(R_{0.86}\right)$ from ASTER observations on 2 December 2004 (i.e., cloud mask test i). (b) Same as (a) but showing the band 5 reflectance $\left(R_{2.1}\right)$ (i.e., cloud mask test ii). (c) Same as (a) but showing the color ratio $r_{1}$ (i.e., cloud mask test iii). (d) Same as (a) but showing the color ratio $r_{2}$ (i.e., cloud mask test iv). (e) Same as (a) but showing the brightness temperature $T_{\mathrm{B}, 11}$ (i.e., cloud mask test v). (f) Same as (a) but showing the cloudiness flags "0"-“3”, after applying cloud mask tests (i)-(v). 
Step 1

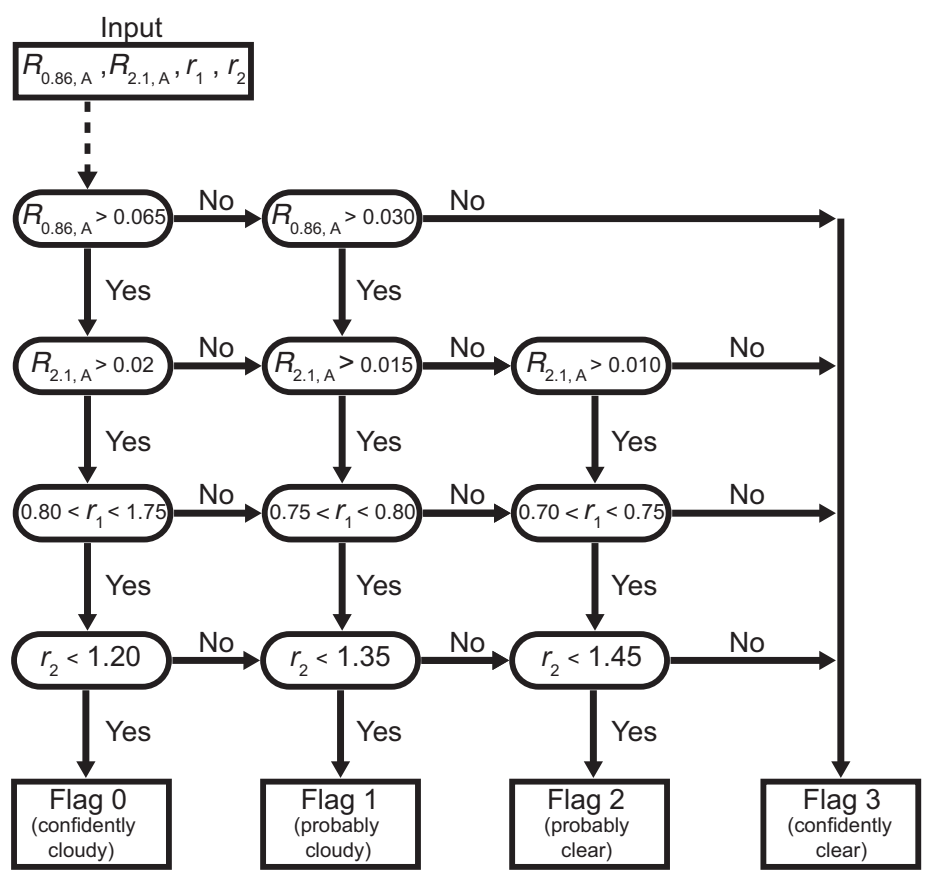

Step 2

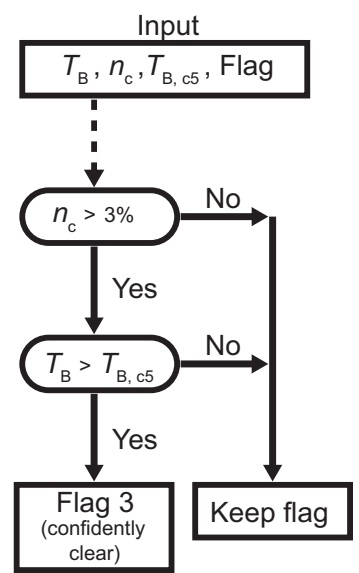

Figure 3. Outline of the new ASTER cloud mask algorithm. Step 1 illustrates the decision tree including cloudiness tests (i)-(iv) based on ASTER band $3 \mathrm{~N}$ and 5 reflectances $R_{0.86}$ and $R_{2.1}$, as well as color ratios $r_{1}$ and $r_{2}$. The results of tests (i)-(iv) yield a designation of cloudiness flag "0" (confidently cloudy), "1" (probably cloudy), "2" (probably clear), or "3" (confidently clear) for each pixel. Step 2 illustrates the correction for complex broken cumulus scenes, as well as cases with pronounced sunglint. This correction, test (v), is based on the derived cloudiness flags from step 1, the brightness temperature $T_{\mathrm{B}, 11}$ (calculated from the ASTER band 14 radiances), the percentage of clear pixels with cloudiness flag " 3 " $\left(n_{\mathrm{c}}\right)$, and the 5 th percentile of $T_{\mathrm{B}, 11}$ sampled over all clear pixels $\left(T_{\mathrm{B}, \mathrm{c} 5}\right)$.

Figure 2a-b show observations of $R_{0.86, \mathrm{~A}}$ and $R_{2.1, \mathrm{~A}}$ over a multitude of small cumuli and the ocean surface. The surface samples exhibit $R_{0.86, \mathrm{~A}} \leq 0.03$ and $R_{2.1, \mathrm{~A}} \leq 0.008$, whereas the thick parts of the cumuli are characterized by $R_{0.86, \mathrm{~A}}>$ 0.1 and $R_{2.1, \mathrm{~A}}>0.015$. Meanwhile, over cloud edges and very thin cloud parts $R_{0.86, \mathrm{~A}}<0.1$ and $R_{2.1, \mathrm{~A}}<0.015$ are observed. Figure 2c-d illustrate $r_{1}$ and $r_{2}$, respectively. As mentioned earlier, these ratios show values around 1 for cloudy pixels, while the ocean can be clearly discriminated with values of $r_{1} \leq 0.7$ and $r_{2} \geq 1.45$. Results for $T_{\mathrm{B}, 11}$, shown in Fig. 2e, illustrate a decrease in derived brightness temperatures for cloudy pixels compared to the ocean surface in the range of (2-3) K. Finally, Fig. $2 f$ shows the derived cloud mask for the example case sampled on 22 January 2005 , yielding reliable results compared to the observations of $R_{0.86, \mathrm{~A}}$.

A comparison between calculated $C_{\mathrm{A}}$ based on the cloud masking scheme reported in Zhao and Di Girolamo (2006), which utilizes a single case-by-case threshold for the digital counts $d_{\mathrm{A}}$ sampled in the ASTER VNIR band, and those based on cloudiness tests (i)-(v) shows a high agreement. A frequency distribution of the difference in scene cloud covers between the case-by-case threshold and the new cloud masking scheme $\left(\Delta C_{\mathrm{A}}\right)$ is shown in Fig. 4. Derived $\Delta C_{\mathrm{A}}$ is in the range of $\Delta C_{\mathrm{A}}=-0.07-0.10$, with a median difference amounting to an underestimation of about 0.004 and an interquartile range (IQR) of 0.019 . These maximum deviations, however, are only observed for a small number of cases. These are characterized by either strong sunglint, which makes it difficult to reliably detect all clouds with just a single threshold for $R_{0.86, \mathrm{~A}}$, or by a complex cloud structure with pronounced horizontal photon transport, which yields some false cloudy pixel designations by the singlethreshold scheme. For these cases cloudiness test (v) assures that the new ASTER cloud mask algorithm produces more reliable results. The majority of scenes $(90.4 \%)$ are characterized by a good agreement in estimated cloud amount in the range of $-0.04 \leq \Delta C_{\mathrm{A}} \leq 0.04$. The slight skew towards positive $\Delta C_{\mathrm{A}}$ values is consistent with the cloud conservative goal of the new automated algorithm for the purpose of this study. On the pixel level it is found that of all cloudy pixels, as determined by the threshold introduced in Zhao and Di Girolamo (2006), $80.8 \%$ are also identified by the new cloud masking scheme, about $14.6 \%$ are missed but have no successful cloud property retrieval, $0.03 \%$ are missed and have a retrieved cloud optical thickness $\tau_{\mathrm{A}} \geq 5$, and $4.6 \%$ are missed and exhibit $\tau_{\mathrm{A}}<5$. Of all clear pixels, as determined by the single-band threshold, $99.4 \%$ are also identified as 


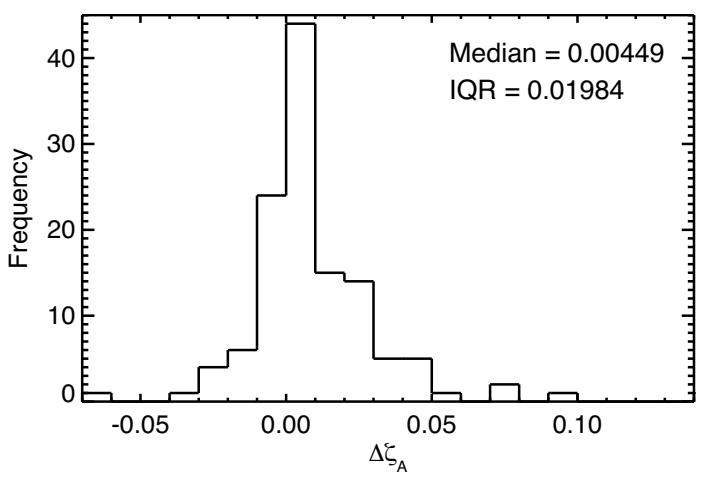

Figure 4. Difference in domain-averaged cloud cover $\left(\Delta C_{\mathrm{A}}\right)$ between the single-band threshold reported by Zhao and Di Girolamo (2006) and the new ASTER cloud masking scheme. Data were sampled over 124 broken cumulus scenes in the tropical southern Atlantic Ocean. Values for the median and interquartile range (IQR) are given.

clear by the new cloud masking scheme, $0.2 \%$ are characterized as cloudy with a failed cloud property retrieval, and $0.4 \%$ exhibit a cloudy designation and $\tau_{\mathrm{A}}<1$.

\subsection{Retrieval algorithm}

After cloud masking a retrieval of cloud top, optical, and microphysical properties is performed. The research-level ASTER retrieval setup uses the same algorithms as the operational MODIS C6 retrieval, which has been extensively tested and documented.

\subsubsection{Cloud top properties}

The retrievals of ASTER cloud top pressure, cloud top temperature and cloud top height are performed using the optimal estimation method in conjunction with the operational MODIS C6 IR window retrieval. This precise algorithm combination is used with great success for the operational retrievals of cloud top properties for the MSG SEVIRI imager (Hamann et al., 2014). Data input is provided by the collected radiances $I_{\mathrm{A}}$ in combination with the profiles of atmospheric temperature, moisture, ozone, and surface temperature. The current implementation of the ASTER retrieval uses Global Data Assimilation System (GDAS) $1^{\circ}$ analysis from the National Centers for Environmental Prediction (NCEP) for this purpose (Derber et al., 1991). The surface emissivity data come from the broadband spectral emissivity database produced for the MOD07 atmospheric profiles product (Seemann et al., 2008). To account for the presence of possible snow or sea ice in the scene the NCEP sea ice product (Hunke and Dukowicz, 1997) is used together with the National Snow and Ice Data Center (NSIDC) $27 \mathrm{~km}$ resolution 5-day running average land snow cover (Nolin et al., 1998). The retrieval begins by obtaining the profiles of IR transmittance and radiance for the given ancil- lary atmospheric and surface parameters at the specific pixel. The calculations are performed using the Pressure-layer Fast Algorithm for Atmospheric Transmittance (PFAAST) code (Strow et al., 2003). PFAAST is also implemented in the operational MODIS cloud top properties retrieval algorithm documented in Baum et al. (2012), except for the ASTER retrievals, for which the full ASTER SRFs are used instead of MODIS ones. The cloud thermodynamic phase is subsequently computed using the bispectral IR method based on the brightness temperature difference between the 8.5 and $11 \mu \mathrm{m}$ bands. The method is identical to the one used by the operational MODIS C5.1 IR cloud thermodynamic phase retrieval (Baum et al., 2000). After determining the thermodynamic phase, the retrievals of cloud top pressure, cloud top temperature, and cloud top altitude are performed assuming unity cloud emissivity as an initial guess. Actual values are derived from the optimal estimation algorithm, which is also used by the MODIS Visible Infrared Imaging Radiometer Suite (VIIRS) data continuity product for cloud top properties (Heidinger et al., 2014). If the calculated cloud top pressure is larger than $650 \mathrm{mb}$ the operational MODIS C6 IR window retrieval algorithm is used to calculate the final value of cloud top pressure (Baum et al., 2012). Cloud phase is also corrected as necessary based on cloud top temperature and cloud top pressure provided by the optimal estimation algorithm. If prior to the optimal estimation calculations the cloud phase was identified as liquid water, but the cloud top temperature is less than $245 \mathrm{~K}$ or cloud top pressure is less than $375 \mathrm{mb}$, the cloud phase value is changed to ice.

\subsubsection{Cloud optical and microphysical properties}

The retrievals of cloud optical thickness $\tau_{\mathrm{A}}$ and effective droplet radius $r_{\text {eff,A }}$ are based on the bispectral retrieval approach, which applies atmospherically corrected cloud top reflectances at two distinct wavelength bands and utilizes retrieval lookup tables (LUT) (Twomey and Seton, 1980; Nakajima and King, 1990; Rossow and Schiffer, 1991). This approach uses the distinct sensitivities of reflectances in the VNIR to $\tau$ and reflectances in the SWIR to $r_{\text {eff }}$ (Marshak et al., 2006). ASTER bands $3 \mathrm{~N}$ and 5 provide the VNIR and SWIR reflectances, respectively. Similar to the retrieval of cloud top properties, the ASTER retrieval uses the same algorithms as the operational MODIS C6 retrievals described in King et al. (1997), Platnick et al. (2003), and MODIS Characterization Support Team (2012).

Atmospheric correction, which is a function of cloud top height, is performed by generating two-way atmospheric transmittance tables containing the effects of water vapor and molecular absorption by various gases (Platnick et al., 2003; Wind et al., 2010). Simulations are done with MODTRAN code version 4.2r1 (Berk et al., 1998) for the complete ASTER VNIR and SWIR range (considering the full SRF of each band). The standard atmosphere in the MODTRAN input is modulated by the averaged clear-sky profiles from 
the European Centre for Medium-Range Weather Forecasts (ECMWF) reanalysis (ERA-40) database (Chevallier, 2002). Band 2 and $3 \mathrm{~N}$ reflectances require a correction for abovecloud ozone amount following the method described in Platnick et al. (2003) for the operational MODIS C6 retrieval algorithm. Here, the below-cloud ozone amount is assumed to be negligible and the total column ozone variable of the NCEP GDAS is used as input. Once all corrections are applied, the surface contribution is removed from the measured ASTER reflectance. For that purpose the gap-filled MODIS surface albedo product is used (Moody et al., 2005, 2007, 2008) for retrievals over land. When retrievals are performed over ocean, the NCEP GDAS variables U10M and V10M are used to derive the value of wind speed. This wind speed is used as input in the parameterization following Cox and Munk (1954a, b), which yields the wind speed-dependent bidirectional ocean surface reflectance. Similar to the corrections in the cloud top retrievals, the NSIDC land snow cover and NCEP sea ice products are used to account for the presence of snow or sea ice in the land albedo and ocean surface reflectance. The estimated snow and ice fractions, together with the statistical ecosystem-based MODIS spectral snow and ice albedo product (Moody et al., 2007) and ecosystem type from the International Geosphere-Biosphere Programme (IGBP) data set (Loveland et al., 2000), provide the means to estimate the final value of surface albedo.

The interpolation of the VNIR and SWIR reflectances is performed in different LUTs to accommodate the differences in the band centers and SRFs between ASTER and MODIS (see Fig. 1). LUTs were generated with the discrete ordinates radiative transfer (DISORT) model developed by Stamnes et al. $(1988,2000)$, and the computations were carried out with 64 streams to capture both upwelling and downwelling radiance. Similar to the correction of surface contributions, the ocean surface reflectance is obtained from the Cox-Munk parameterization, as implemented in the radiative transfer library libRadtran (Mayer and Kylling, 2005; Mayer, 2009). The single-scattering properties of liquid water clouds were computed from Mie theory according to Wiscombe (1980), assuming a modified gamma droplet size distribution with an effective variance of 0.10 . The LUTs do not include the additional contributions from Rayleigh scattering, which are added to the atmospherically corrected ASTER reflectances before a retrieval is attempted. The added amount of Rayleigh scattering is a function of cloud top pressure and is accounted for dynamically, using the retrieved value of cloud top pressure as described in Wang and King (1997). For both MODIS and ASTER, the retrieved $\tau$ is scaled to the respective $0.65 \mu \mathrm{m}$ band (i.e., band 1 for MODIS and band 2 for ASTER).

It must be noted that for the cloud property retrieval at $15 \mathrm{~m}$ horizontal resolution each SWIR reflectance sample at $30 \mathrm{~m}$ resolution is replicated onto 4 subpixels to match the band $3 \mathrm{~N}$ resolution. This introduces uncertainties in the retrieved cloud parameters at the highest ASTER resolution.
As described in Sect. 5.4 these uncertainties are estimated to be \pm 0.5 (for $\tau_{\mathrm{A}}$ ) and $\pm 0.7 \mu \mathrm{m}$ (for $r_{\text {eff,A }}$ ).

King et al. (1997) and Platnick et al. (2004) discussed the retrieval uncertainties associated with MODIS cloud products, which are the result of instrument errors, uncertainties in the radiometric calibrations and the applied radiative transfer model, and ancillary data sets used as input for the atmospheric correction algorithm, among other components. The current MODIS retrieval products provide pixel-level uncertainty estimates for $\tau$ and $r_{\text {eff. Because the ASTER re- }}$ trieval algorithm deploys the same retrieval code, ASTER pixel-level retrieval uncertainties are derived in a similar way. They are comprised of uncertainties in the applied surface albedo (15\%), calibration and model uncertainties (5\%), and uncertainties in the amount of above-cloud precipitable water, which is an input variable in the atmospheric correction $(20 \%)$.

An approximate uncertainty range due to radiometric uncertainties only can be estimated by applying the individual measurement uncertainties $\delta$ of the simulated reflectances $R_{\mathrm{L}}$ in the VNIR and SWIR by

$$
\begin{aligned}
& \Delta \tau_{\mathrm{L}}\left(R_{\mathrm{L}}\right)=\frac{\tau_{\mathrm{L}}\left(R_{\mathrm{L}}\right)-\tau_{\mathrm{L}}\left(R_{\mathrm{L}} \pm \delta\right)}{\tau_{\mathrm{L}}\left(R_{\mathrm{L}}\right)} \\
& \Delta r_{\mathrm{eff}, \mathrm{L}}\left(R_{\mathrm{L}}\right)=\frac{r_{\text {eff, } \mathrm{L}}\left(R_{\mathrm{L}}\right)-r_{\mathrm{eff}, \mathrm{L}}\left(R_{\mathrm{L}} \pm \delta\right)}{r_{\mathrm{eff}, \mathrm{L}}\left(R_{\mathrm{L}}\right)} .
\end{aligned}
$$

Here, $\delta$ can either increase or decrease the actually observed $R_{\mathrm{L}}$. Calculating $\Delta \tau_{\mathrm{L}}$ and $\Delta r_{\mathrm{eff}, \mathrm{L}}$ for each possible combination of $R_{\mathrm{L}} \pm \delta$ in the VNIR and SWIR yields an expected uncertainty range for the retrieved cloud properties. Assuming $\delta<4 \%$ (Yamaguchi et al., 1998) and including results of $r_{\text {eff,L }}>4 \mu \mathrm{m}$ and $\tau_{\mathrm{L}}>4$ only yields mean retrieval uncertainties of $\Delta \tau_{\mathrm{L}}=0.15$ and $\Delta r_{\text {eff, } \mathrm{L}}=0.23$, respectively.

\subsection{LUT differences due to SRF differences}

The discussion in Sect. 2.3 showed that there are differences between the VNIR and SWIR SRFs of ASTER and MODIS, which requires the calculation of ASTER-specific LUTs where the spectral scattering properties (i.e., extinction coefficient, single-scattering albedo, and scattering phase function) are integrated over the ASTER SRFs. These new LUTs subsequently provide the means to retrieve the cloud optical thickness $\tau_{\mathrm{A}}$ and $r_{\mathrm{eff}, \mathrm{A}}$ from sampled ASTER VNIR and SWIR reflectances $R_{0.86, \text { LUT,A }}$ and $R_{2.1, \text { LUT,A }}$.

Figure 5a illustrates an example for ASTER (solid black lines) and MODIS (dash-dotted green lines) LUTs, based on 1-D reflectance calculations over a model cloud over the ocean. The solar and sensor zenith angles are $\theta_{0}=57^{\circ}$ and $\theta_{\mathrm{s}}=8.5^{\circ}$, with a relative azimuth angle (defined as the sensor azimuth angle $-180^{\circ}$ - the solar azimuth angle) of $\varphi_{\text {rel }}=5.0^{\circ}$. This input solar and viewing geometry, which is the same for both ASTER and MODIS, is defined as "Geometry 1". The relationship between VNIR reflectance and $\tau_{\mathrm{L}}$ exhibits the well-known monotonically 

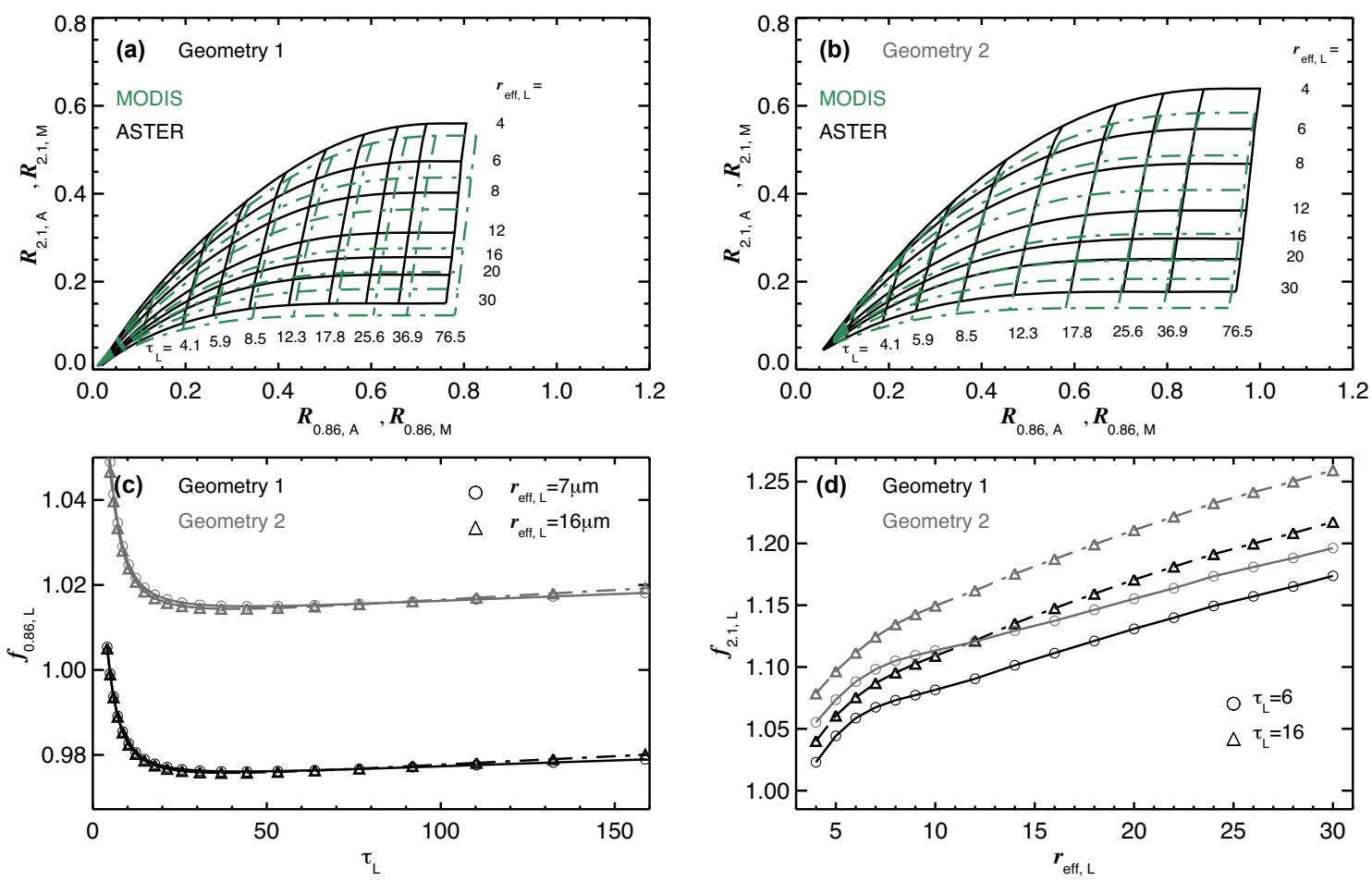

Figure 5. Example lookup table (LUT) for ASTER (solid black lines) and MODIS (dash-dotted green lines) for (a) "Geometry 1" and (b) "Geometry 2". See text for details on the specific solar and viewing geometries. (c) The reflectance ratio $f_{0.86, \mathrm{~L}}$ of ASTER to MODIS VNIR reflectances as a function of cloud optical thickness $\tau_{\mathrm{L}}$ for Geometry 1 (black line) and Geometry 2 (gray line). Simulations for two different values of effective droplet radius $r_{\text {eff, } \mathrm{L}}$ are indicated by different symbols. (d) The reflectance ratio $f_{2.1, \mathrm{~L}}$ of ASTER to MODIS SWIR reflectances as a function of $r_{\mathrm{eff}, \mathrm{L}}$ for Geometry 1 and Geometry 2 . Simulations for two different values of $\tau_{\mathrm{L}}$ are indicated by different symbols.

increasing (concave) behavior for both sensors and generally $R_{0.86, \text { LUT,A }}<R_{0.86, \text { LUT,M }}$ (i.e., for the same cloud optical thickness ASTER appears darker than MODIS). There is a slight decrease in VNIR reflectance with increasing $r_{\text {eff, }}$, which was also stated in Marshak et al. (2006). Conversely, there is a monotonically decreasing (convex) behavior of simulated SWIR reflectances with increasing $r_{\text {eff,L }}$ and $R_{2.1, \text { LUT,A }}>R_{2.1, \mathrm{LUT}, \mathrm{M}}$ (i.e., for the same effective droplet radius ASTER appears brighter than MODIS). A second set of example LUTs is shown in Fig. 5b. Here, the solar and viewing geometry, defined as "Geometry 2", is based on example scene C19, which is discussed in Table 2 and Sect. 4.2. This scene yields $\theta_{0}=20^{\circ}$ and $\varphi_{0}=137^{\circ}$. Sensor zenith and azimuth angles are generally different between ASTER and MODIS, with differences varying from scene to scene. For $\mathrm{C} 19$ the ASTER viewing geometry is characterized by $\theta_{\mathrm{s}}=8.59^{\circ}(\mathrm{VNIR})$ and $\theta_{\mathrm{s}}=8.54^{\circ}$ (SWIR), with a relative azimuth angle close to zero. For MODIS $\theta_{\mathrm{s}}=9.64^{\circ}$ and $\varphi_{\text {rel }}=38^{\circ}$. The decrease in $\theta_{0}$ yields an increase in both VNIR and SWIR reflectances (for both sensors). However, for this geometry both ASTER bands appear brighter than the respective MODIS bands. This means that a comparison of
ASTER and MODIS reflectances depends on the exact viewing geometry of the respective scene.

The ratio $f_{0.86, \mathrm{~L}}$ of simulated ASTER to MODIS VNIR reflectance for each $\left(\tau_{\mathrm{L}}, r_{\mathrm{eff}, \mathrm{L}}\right)$ pair is defined as

$f_{0.86, \mathrm{~L}}\left(\tau_{\mathrm{L}}, r_{\mathrm{eff}, \mathrm{L}}\right)=\frac{R_{0.86, \mathrm{LUT}, \mathrm{A}}\left(\tau_{\mathrm{L}}, r_{\mathrm{eff}, \mathrm{L}}\right)}{R_{0.86, \mathrm{LUT}, \mathrm{M}}\left(\tau_{\mathrm{L}}, r_{\mathrm{eff}, \mathrm{L}}\right)}$.

$f_{0.86, \mathrm{~L}}$ for both geometries and two constant values of input cloud effective droplet radius $r_{\text {eff,L }}$ (highlighted by different symbols) is illustrated in Fig. 5c. Depending on the geometry, $0.97<f_{0.86, \mathrm{~L}}<1.05$ (varying $\theta_{0}$ in the range $1-70^{\circ}$ yields $0.93<f_{0.86, \mathrm{~L}}<1.06$ ). For $\tau_{\mathrm{L}}>10$ the ratio between ASTER and MODIS reflectances becomes almost constant and there is almost no dependence of $f_{0.86, \mathrm{~L}}$ on $r_{\mathrm{eff}, \mathrm{L}}$. The ratio $f_{2.1, \mathrm{~L}}$ of ASTER to MODIS SWIR reflectances is derived similarly to Eq. (4) and shown in Fig. 5d. The shift towards a larger center wavelength for the ASTER SWIR band yields an increase in scattering efficiency and singlescattering albedo. As a result the ASTER SWIR bands appear significantly brighter than the respective MODIS band and the specific solar and viewing geometry only affects the magnitude of $f_{2.1, \mathrm{~L}}$. There is a noticeable dependence of $f_{2.1, \mathrm{~L}}$ on both $\tau_{\mathrm{L}}$ and $r_{\mathrm{eff}, \mathrm{L}}$. Depending on the geometry, 
Table 2. Case number (C1-C48) and sample date of each MBL scene in this study. The date format is MM/DD/YYYY hour:minute:second.

\begin{tabular}{lr|lr|ll}
\hline Case no. & \multicolumn{2}{r}{ Date } & Case no. & Date & Case no. \\
\hline 1 & $03 / 02 / 2006 / 19: 14: 44$ & 21 & $06 / 25 / 2004 / 19: 10: 45$ & 41 & Date \\
2 & $03 / 06 / 2005 / 19: 20: 37$ & 22 & $07 / 04 / 2007 / 19: 09: 35$ & 42 & $10 / 21 / 2006 / 19: 09: 31$ \\
3 & $03 / 06 / 2005 / 19: 20: 46$ & 23 & $07 / 04 / 2007 / 19: 10: 19$ & 43 & $10 / 25 / 2005 / 19: 14: 44$ \\
4 & $03 / 06 / 2005 / 19: 20: 55$ & 24 & $07 / 04 / 2007 / 19: 10: 46$ & 44 & $10 / 25 / 2006 / 18: 45: 26$ \\
5 & $03 / 06 / 2005 / 19: 21: 04$ & 25 & $07 / 11 / 2007 / 19: 16: 06$ & 45 & $10 / 25 / 2006 / 18: 45: 35$ \\
6 & $03 / 06 / 2005 / 19: 21: 13$ & 26 & $07 / 20 / 2007 / 19: 10: 07$ & 46 & $10 / 30 / 2006 / 19: 03: 35$ \\
7 & $03 / 08 / 2005 / 19: 08: 35$ & 27 & $07 / 20 / 2007 / 19: 10: 16$ & 47 & $12 / 03 / 2005 / 19: 20: 56$ \\
8 & $03 / 08 / 2005 / 19: 08: 44$ & 28 & $07 / 20 / 2007 / 19: 10: 25$ & 48 & $12 / 16 / 2004 / 19: 20: 41$ \\
9 & $03 / 08 / 2005 / 19: 08: 53$ & 29 & $08 / 18 / 2006 / 19: 09: 01$ & & \\
10 & $04 / 19 / 2006 / 19: 14: 55$ & 30 & $08 / 18 / 2006 / 19: 09: 18$ & & \\
11 & $04 / 19 / 2006 / 19: 15: 13$ & 31 & $08 / 26 / 2003 / 19: 09: 37$ & & \\
12 & $04 / 19 / 2006 / 19: 15: 22$ & 32 & $08 / 26 / 2003 / 19: 09: 55$ & & \\
13 & $04 / 19 / 2006 / 19: 15: 31$ & 33 & $08 / 26 / 2003 / 19: 10: 12$ & & \\
14 & $05 / 13 / 2003 / 19: 15: 46$ & 34 & $08 / 29 / 2006 / 18: 52: 02$ & & \\
15 & $05 / 30 / 2006 / 19: 08: 57$ & 35 & $08 / 29 / 2006 / 18: 52: 11$ & & \\
16 & $06 / 02 / 2007 / 19: 09: 29$ & 36 & $09 / 02 / 2003 / 19: 15: 12$ & & \\
17 & $06 / 02 / 2007 / 19: 09: 47$ & 37 & $09 / 07 / 2005 / 19: 14: 31$ & & \\
18 & $06 / 03 / 2005 / 19: 14: 42$ & 38 & $09 / 07 / 2005 / 19: 14: 49$ & & \\
19 & $06 / 10 / 2005 / 19: 20: 47$ & 39 & $09 / 10 / 2006 / 19: 15: 21$ & & \\
20 & $06 / 10 / 2005 / 19: 21: 04$ & 40 & $09 / 11 / 2004 / 19: 21: 08$ & \\
\hline
\end{tabular}

$1.02<f_{2.1, \mathrm{~L}}<1.25$ (varying $\theta_{0}$ in the range $1-70^{\circ}$ yields $\left.0.98<f_{0.86, \mathrm{~L}}<1.28\right)$.

The calculation of the ratios $f_{0.86, \mathrm{~L}}$ and $f_{2.1, \mathrm{~L}}$ allows for a direct comparison of measured ASTER and MODIS reflectances, as illustrated in Sects. 5.2.1 and 5.3.2.

\section{Examples of high-resolution retrievals}

This section introduces all ASTER MBL scenes used in this study. Moreover, examples of the retrieved cloud optical thickness and effective droplet radius based on highresolution ASTER reflectance measurements are presented.

\subsection{Data set}

Since the goal of this study is to examine the feasibility of ASTER cloud property retrievals in comparison to MODIS, a sufficient number of samples of both fully and partially cloudy pixels at $1000 \mathrm{~m}$ scales is required. For this reason, not all of the 210 ASTER MBL scenes that were used in evaluating the $15 \mathrm{~m}$ cloud mask in Sect. 3.1 are sufficient. The scenes sampled in the tropical western Atlantic (Zhao and Di Girolamo, 2006) were populated entirely by trade wind cumuli with a peak in the cloud fraction distribution at 400$500 \mathrm{~m}$ in cloud equivalent diameters (see Zhao and Di Girolamo, 2007). The data set used in the following comparison consists of $48 \mathrm{MBL}$ scenes sampled over the Pacific Ocean off the coast of California between May 2003 and July 2007. Granules were manually chosen to include MBL clouds that resemble altocumulus or broken cumulus scenes and thus are characterized by extensive MBL cloud cover and cloud sizes. The number of available cases is constrained by the availability of co-located ASTER and MODIS data with successful cloud property retrievals at a horizontal resolution of $1000 \mathrm{~m}$ (which excludes some broken cumulus scenes). Moreover, selected scenes are characterized by the absence of overlying cirrus, complex multi-layered cloud systems, and pixels with ice phase. It was made sure that the cases sampled in 2007 are not affected by the reduced dynamic range of the ASTER SWIR band signal, which started to affect the ASTER data starting mid-2007. The area covered by the 48 MBL scenes is embedded within $125.924-117.038^{\circ} \mathrm{W}$ and $32.051-44.427^{\circ} \mathrm{N}$

Table 2 lists the case numbers $(\mathrm{C} 1-\mathrm{C} 48)$, as well as the sample date of each scene. A wide range of different scene characteristics are covered, with estimated domain-averaged cloud covers $C_{\mathrm{M}}$, based on MODIS cloud flags " 0 " and "1" (i.e., "confidently" and "probably cloudy" pixels), between $C_{\mathrm{M}}=0.01$ and 1.00 . There are 25 scenes with $C_{\mathrm{M}}=$ $0.75-0.99$, 9 scenes with $C_{\mathrm{M}}=0.25-0.74$, and 9 scenes with $C_{\mathrm{M}}<0.25$. Completely overcast conditions (i.e., $C_{\mathrm{M}}=$ $1.00)$ are found for 18 scenes. The solar zenith angle for these cases varies between $\theta_{0}=17.96$ and $63.84^{\circ}$, with 24,34 , and 4 scenes having $\theta_{0}<30^{\circ}, 30^{\circ} \geq \theta_{0}<60^{\circ}$, and $\theta_{0} \geq 60^{\circ}$, respectively.

\subsection{High-resolution retrievals}

Two ASTER scenes are selected as case studies to demonstrate the feasibility of high-resolution ASTER retrievals and 

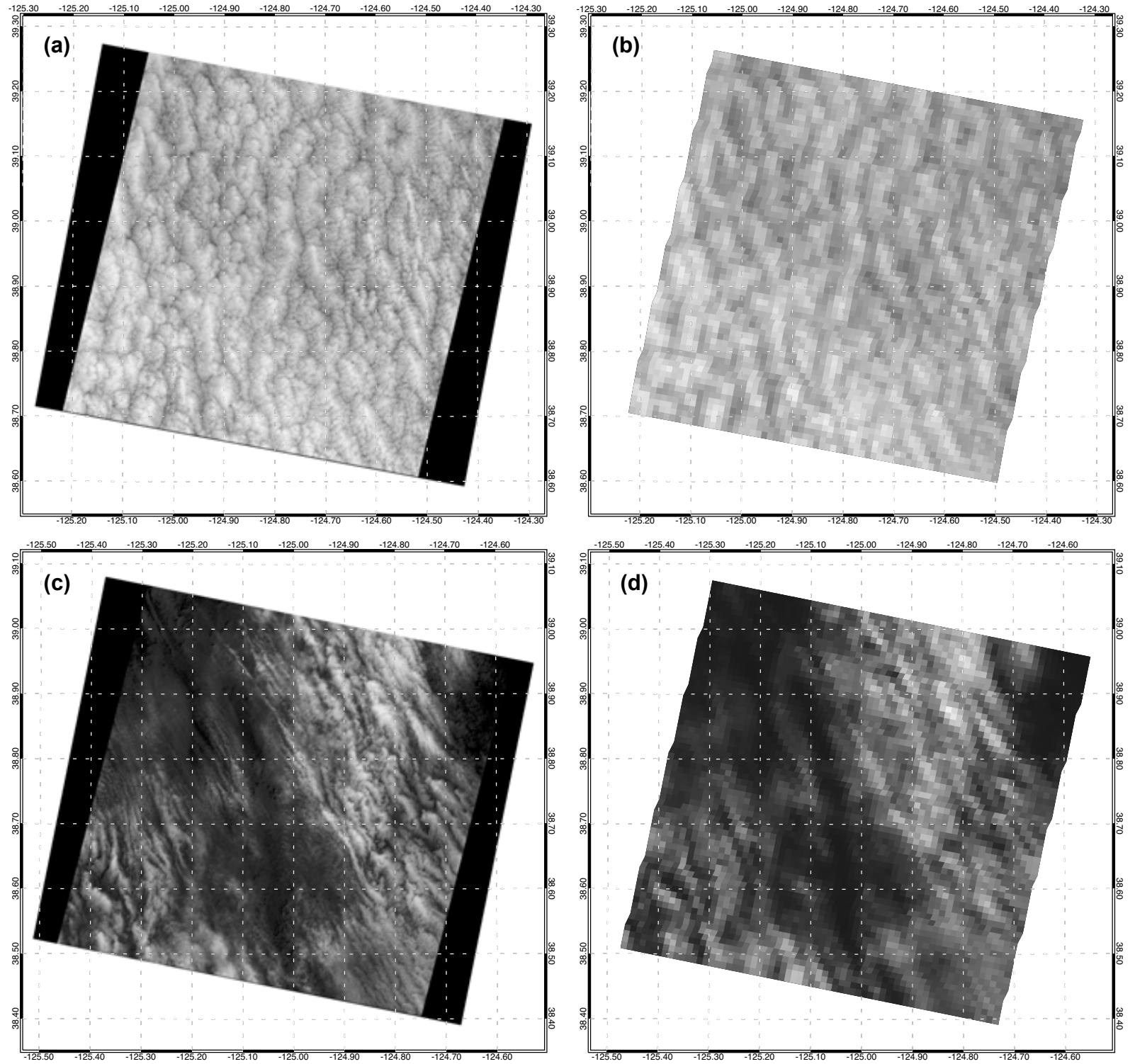

Figure 6. (a) Single-band grayscale image of band 3N reflectances sampled by ASTER on 13 May 2003 off the coast of California (scene C14). (b) Same as (a) but from band 2 reflectances sampled by MODIS. (c)-(d) Same as (a)-(b) but sampled on 10 June 2005 (scene C19).

to highlight the differences between the co-located ASTER and MODIS retrieval products.

The first scene (C14) is an altocumulus field with a domain-averaged cloud cover of $C_{\mathrm{M}}=1.0$ sampled at 19:15 UTC on 13 May 2003. The solar geometry is characterized by $\theta_{0}=24^{\circ}$ and $\varphi_{0}=143^{\circ}$, placing the Sun's position in the southwest of the scene. Figure $6 \mathrm{a}$ shows the singleband grayscale image of $R_{0.86, \mathrm{~A}} \cdot R_{0.86, \mathrm{~A}}$ was sampled with a horizontal resolution of $15 \mathrm{~m}$, which allows for the detection of small-scale cloud inhomogeneities and dynamically induced, cell-like cloud structures. In contrast, the singleband grayscale image of MODIS VNIR reflectances $R_{0.86, \mathrm{M}}$, shown in Fig. 6b, appears visibly smoother due to the horizontal resolution of the measurements of $1000 \mathrm{~m}$.

A second, significantly more inhomogeneous scene (C19) with $C_{\mathrm{M}}=0.88$ is shown in Fig. $6 \mathrm{c}-\mathrm{d}$, illustrating singleband grayscale images of $R_{0.86, \mathrm{~A}}$ and $R_{0.86, \mathrm{M}}$, respectively. C19 was sampled at 19:20 UTC on 10 June 2005. The solar and viewing geometry is similar to C14 with $\theta_{0}=20^{\circ}$ and $\varphi_{0}=136^{\circ}$. The cloud field is characterized by an increased heterogeneity and, while the cloud cover is rather high, larger areas containing thin cloud pixels are visible throughout the scene. Contrary to the ASTER measurements, $R_{0.86, \mathrm{M}}$ for the rather thin parts in the middle of the granule seems to be very low and the numerous delicate cloud structures (e.g., between 

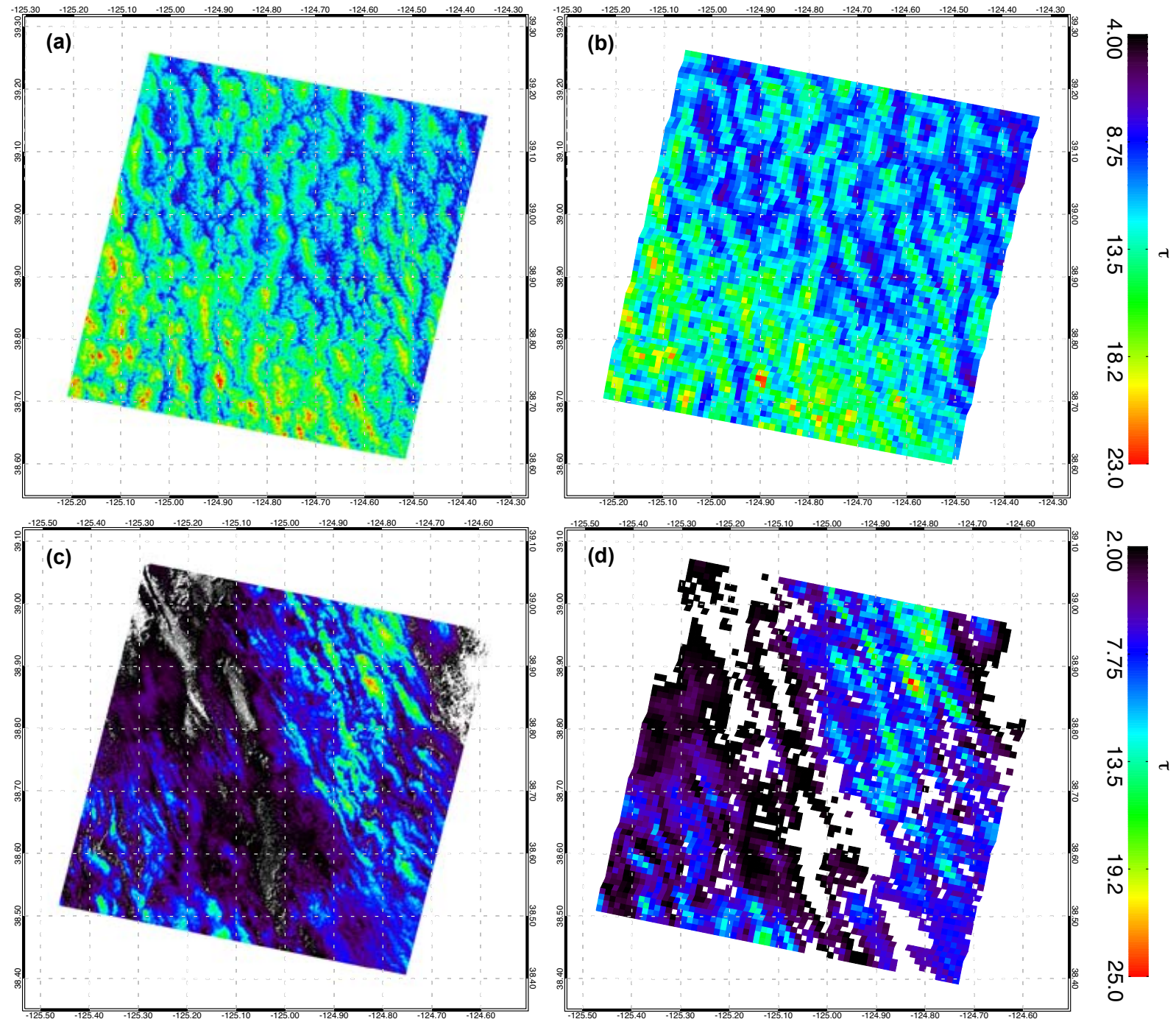

Figure 7. (a) Map of retrieved cloud optical thickness from reflectances sampled by ASTER $\left(\tau_{\mathrm{A}}\right)$ on 13 May 2003 (scene C14). (b) Same as (a) but showing the operational MODIS retrieved cloud optical thickness $\left(\tau_{M}\right)$. (c)-(d) Same as (a)-(b) but for observations on 10 June 2005 (scene C19). The corresponding single-band grayscale images of ASTER band 3N and MODIS band 2 reflectances are shown in Fig. 6a-d.

$125.400-125.100^{\circ} \mathrm{W}$ and $38.700-38.900^{\circ} \mathrm{N}$ ) are smoothed out.

Figure 7a-b show the cloud optical thickness retrieved from $\operatorname{ASTER}\left(\tau_{\mathrm{A}}\right)$ and MODIS $\left(\tau_{\mathrm{M}}\right)$ reflectances sampled above scene $\mathrm{C} 14$ on 13 May 2003. The presented MODIS results include partially cloudy pixels. Observed $\tau_{\mathrm{A}}$ are about 15-18 for the thick cloud parts and dip to around 8 between the cell structures. For the brightest cloud sections $\tau_{\mathrm{A}}$ reaches values of 23. Similar observations can be made from the MODIS retrieval, with $\tau_{\mathrm{M}}=15-18$ for the thicker cloud parts and reduced $\tau_{\mathrm{M}} \approx 8$ for the intermittent sections between the cell structures. As with the ASTER retrievals, there are occasional observations of $\tau_{\mathrm{M}}>20$ for the thickest cloud parts in the south of the granule. However, the rather interesting, fuzzy behavior of $\tau_{\mathrm{A}}$ around individual cells, es- pecially visible in the northeast of the granule, is smoothed by the MODIS observations.

Retrieval results for $\mathrm{C} 19$ are illustrated in Fig. 7c-d, showing $\tau_{\mathrm{A}}$ and $\tau_{\mathrm{M}}$, respectively. A significant number of cloud holes are embedded within larger areas of thin cloudy pixels, where the optical thickness observations can be as low as $\tau_{\mathrm{A}}=2$. Thicker cloud parts include a number of samples with $\tau_{\mathrm{A}} \approx 15$, reaching values of about $\tau_{\mathrm{A}}=25$ at its brightest points. Observed $\tau_{\mathrm{M}}$ for this scene is again comparable to the ASTER results, although there are visibly more MODIS pixels throughout the granule where the retrieval fails. These pixels are characterized by $R_{0.86, \mathrm{M}}$ and $R_{2.1, \mathrm{M}}$ (sampled at $1000 \mathrm{~m}$ ) that are too low for a successful cloud property retrieval (i.e., measurements fall outside the LUT). 

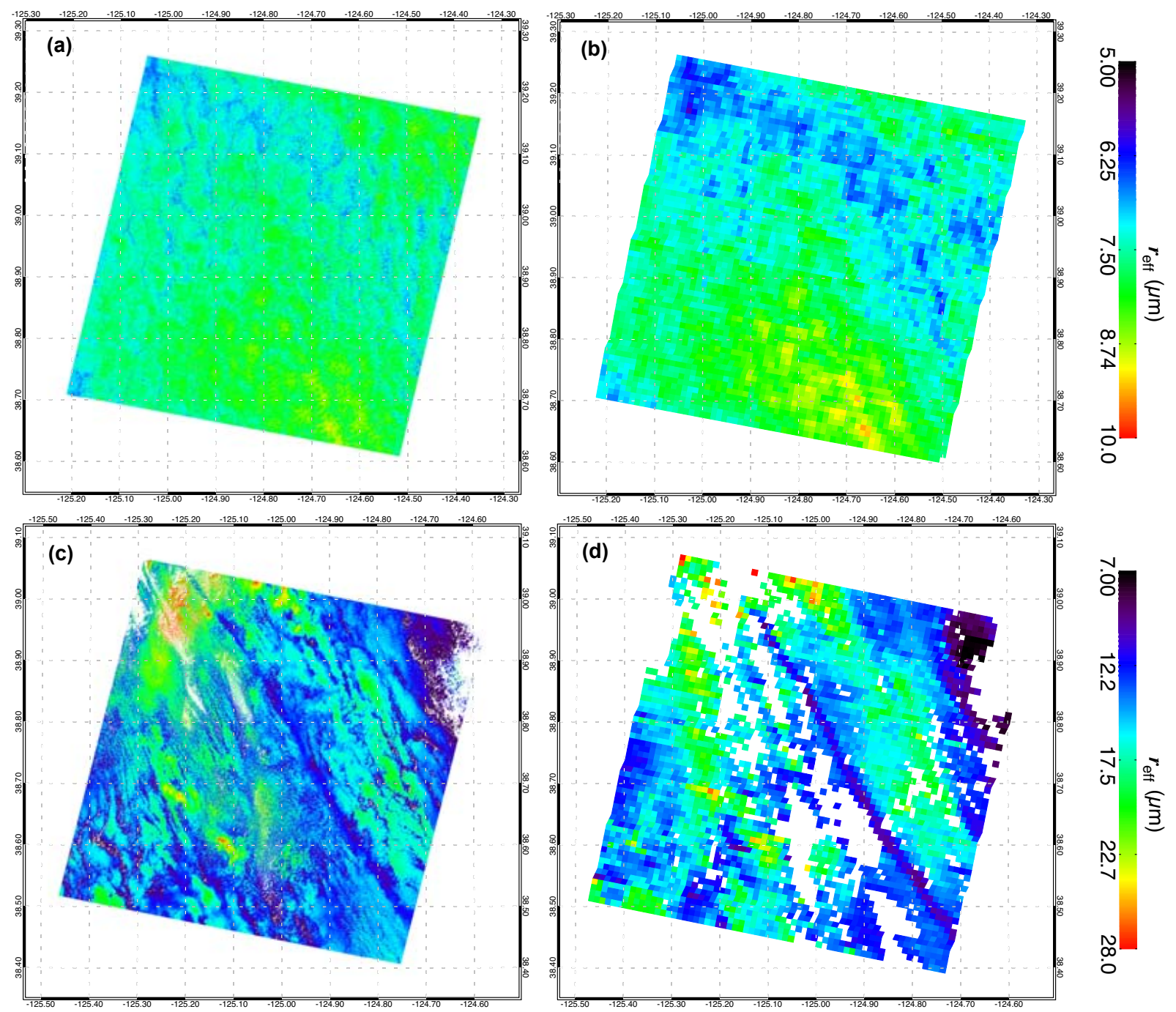

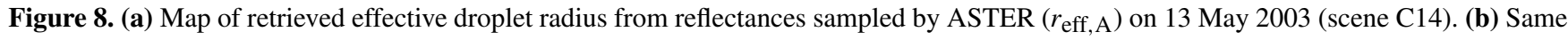
as (a) but showing the operational MODIS retrieved effective droplet radius $\left(r_{\mathrm{eff}, \mathrm{M}}\right)$. (c)-(d) Same as (a)-(b) but for observations on 10 June 2005 (scene C19). The corresponding single-band grayscale images of ASTER band 3N and MODIS band 2 reflectances are shown in Fig. 6a-d.

Results of the effective droplet radius retrieval from ASTER $\left(r_{\text {eff,A }}\right)$ and MODIS data $\left(r_{\text {eff, }}\right)$ are illustrated in Fig. 8. For the example scene C14, shown in Fig. 8a-b, the effective radius retrieval shows a very homogeneous distribution, with the majority of observations around $r_{\mathrm{eff}, \mathrm{A}}=7$ $9 \mu \mathrm{m}$, which is close to a monodisperse $r_{\text {eff, A field. Like- }}$ wise, the MODIS retrieval shows that most results are within $r_{\text {eff, }} \mathrm{M}=6-9 \mu \mathrm{m}$, revealing a good agreement between both sensors. However, there is also visible striping in the $r_{\text {eff, }}$ results, which is caused by electronic crosstalk between various MODIS bands (Xiong et al., 2003, 2009; Sun et al., 2010, 2014).

Figure 8c-d illustrate retrieved $r_{\text {eff, A }}$ and $r_{\text {eff, }}$ for $\mathrm{C} 19$. Similar to the $\tau_{\mathrm{A}}$ and $\tau_{\mathrm{M}}$ results there is a high degree of heterogeneity in observed effective droplet radii, which exhibit a range of $r_{\text {eff,A }}=7-28 \mu \mathrm{m}$. Some of the largest $r_{\text {eff, A }}>20 \mu \mathrm{m}$ are sampled around the thinnest cloud parts, as well as cloud holes, in the northwest of the granule (around $39.00^{\circ} \mathrm{N}$ and $125.2^{\circ} \mathrm{W}$ ). This implies a possible impact of clear-sky contamination and 3-D radiative effects. A similar behavior is observed in the $r_{\text {eff,M }}$ field, although the number of failed retrievals is significantly higher compared to the ASTER data. The delicate cloud structures throughout the scene are characterized by small-scale fluctuations in $r_{\mathrm{eff}, \mathrm{A}}$ between $r_{\text {eff, } \mathrm{A}}=10$ and $20 \mu \mathrm{m}$. The smaller horizontal resolution of the MODIS observations does not capture these finer cloud structures. 

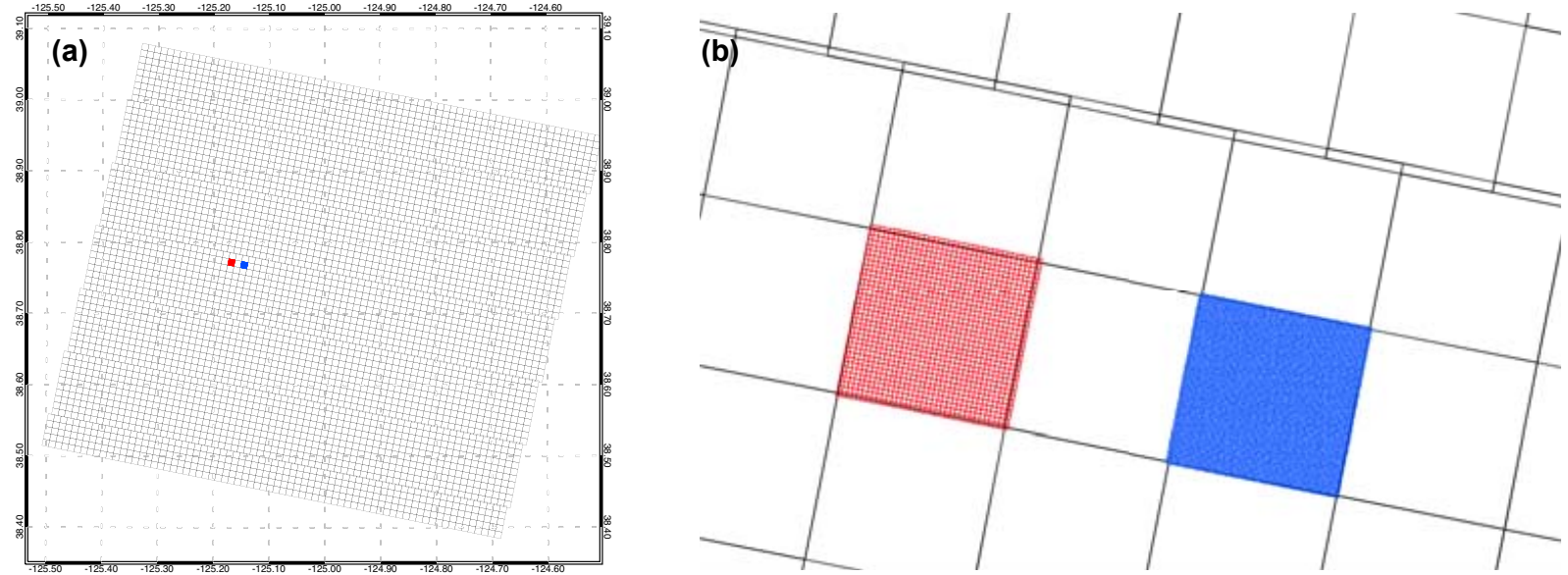

Figure 9. (a) Derived MODIS pixels (gray lines) for the MBL scene observed on 10 June 2005 (C19). For two individual MODIS pixels all co-located ASTER pixels in the VNIR (blue lines) and SWIR (red lines) are shown, which are characterized by a horizontal resolution of 15 and $30 \mathrm{~m}$, respectively. (b) Closeup of the two MODIS pixels.

\section{Comparison of ASTER and MODIS results}

In this section a statistical comparison between the operational MODIS C6 retrieval products with co-located ASTER results is presented, first for the two case studies introduced in Sect. 4.2 and subsequently on a statistical basis for all $48 \mathrm{MBL}$ cloud scenes. By employing the operational MODIS C6 retrieval algorithms, uncertainties in the comparison of retrieved cloud products from both sensors are mitigated. This allows for a comprehensive comparison between the MODIS and ASTER results without biases due to the applied set of equations.

\subsection{Aggregation}

To yield a true comparison of reflectances and retrieved cloud variables, the high-resolution ASTER digital counts $d_{\mathrm{A}}(\Delta \lambda)$ are aggregated within each $1000 \times 1000 \mathrm{~m}$ MODIS pixel. This requires the definition of pixel corners for each ASTER and MODIS observation. In a first step, high-resolution ASTER geolocation information is derived by interpolating the geometric correction tables that are included as $11 \times 11$ arrays in the structural metadata of each ASTER data container. This yields latitude and longitude values for each individual sample and band. Subsequently, the four corners of each ASTER pixel are defined by triangulation between the neighboring geolocation data points. Similar analysis provides the respective corners of each MODIS pixel.

Figure 9a illustrates the derived pixel dimensions (gray lines) for all MODIS observations from scene C19. For two example MODIS pixels, all co-located ASTER VNIR (blue lines) and SWIR (red lines) pixels are shown. A closeup of these two MODIS pixels is given in Fig. 9b. It must be noted that while MODIS pixels from scene $\mathrm{C} 19$ can almost be considered squares, this is not universally true for all MODIS pixels. Scenes closer to the edge of a MODIS swath are characterized by an increase in pixel size along the scan direction.

For the aggregation of digital ASTER counts $d_{\mathrm{A}}(\Delta \lambda)$ within a MODIS pixel, an ASTER sample is included if any of its four corners lies within a respective MODIS pixel. Taking into account the different spatial resolutions of both instruments ( $1000 \mathrm{~m}$ for MODIS, $15 \mathrm{~m}$ for the ASTER VNIR band, $30 \mathrm{~m}$ for the ASTER SWIR band), $d_{\mathrm{A}}(\Delta \lambda)$ from over 4400 and 1100 individual ASTER VNIR and SWIR pixels is aggregated within each MODIS pixel, respectively. It is important to note that $d_{\mathrm{A}}(\Delta \lambda)$ from ASTER samples at the edge of the respective MODIS pixel, which are only partially within a MODIS pixel's boundaries, is not weighted according to the covered area. This yields uncertainties in the aggregated digital counts of $<0.05$. Also, the grayscale images in Fig. 6a and c reveal that there are a number of rows of samples with $d_{\mathrm{A}}(\Delta \lambda)=0$ at the left and right edges (i.e., west and east) of the ASTER domain. The same is true for the upper and lower edges (i.e., north and south), although significantly less samples are affected. MODIS pixels including any of these edge pixels (at the native ASTER resolution) are omitted from the analysis.

The aggregated digital counts are subsequently used to derive aggregated VNIR and SWIR ASTER reflectances $R_{0.86 \text {,aA }}$ and $R_{2.1, \mathrm{aA}}$, which provide the input for the cloud property retrieval. This yields ASTER cloud optical thicknesses $\tau_{\mathrm{aA}}$, effective droplet radii $r_{\mathrm{eff}}$,aA, and atmospherically corrected VNIR and SWIR reflectances $\widehat{R}_{0.86 \text {, aA }}$ and $\widehat{R}_{2.1, \text { aA }}$. Here, the subscript "aA" refers to "ASTER aggregated in MODIS". 

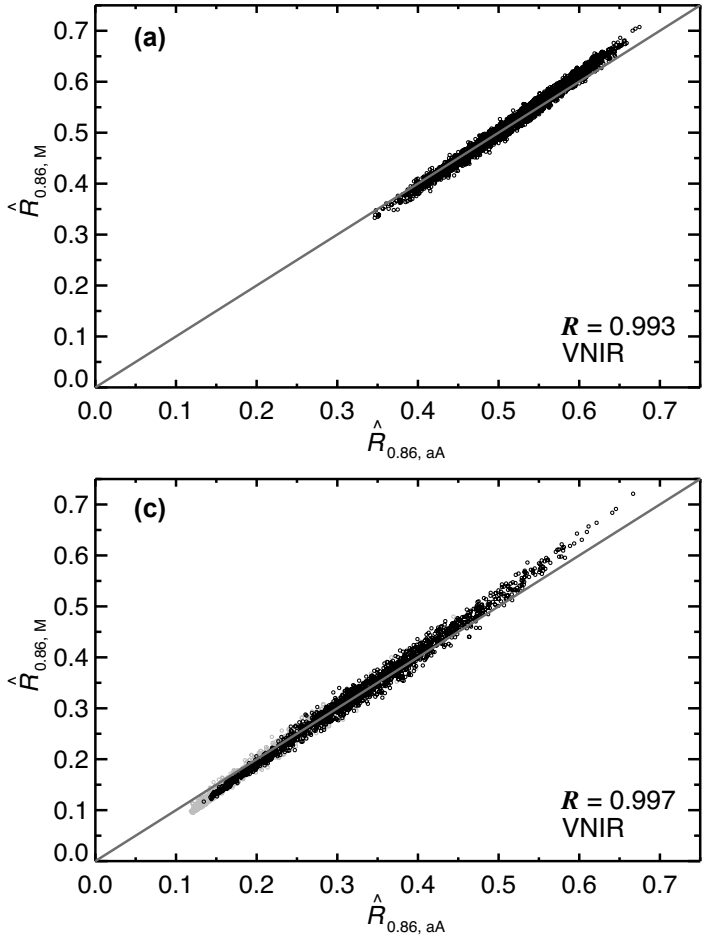
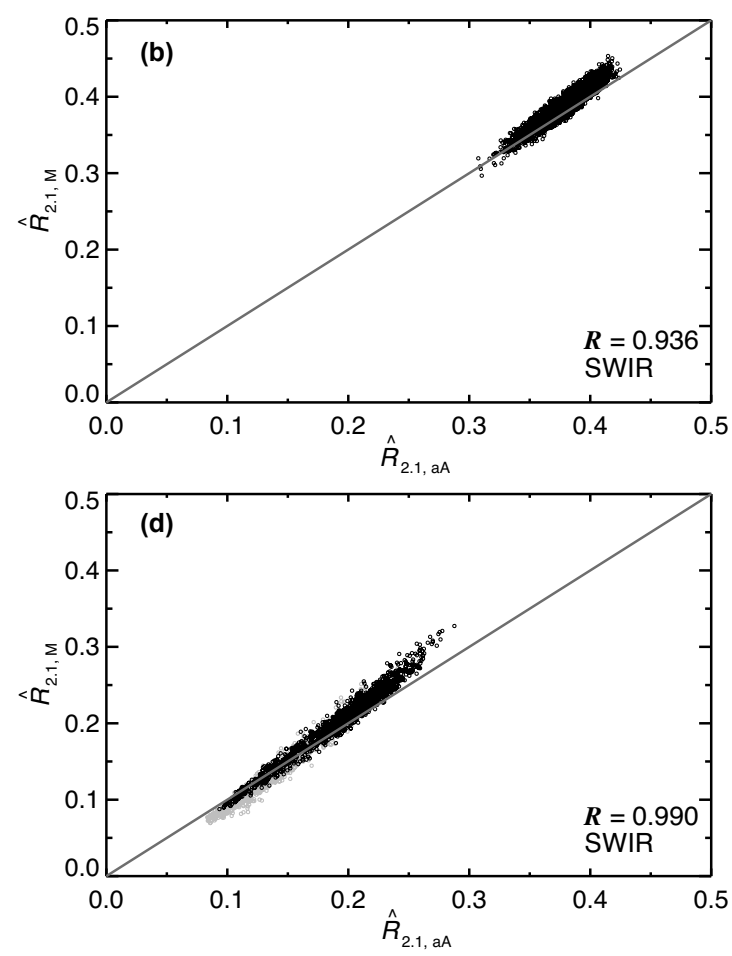

Figure 10. (a) Scatter plot of atmospherically corrected MODIS reflectances $\left(\widehat{R}_{0.86, \mathrm{M}}\right)$ in the VNIR as a function of co-located, atmospherically corrected ASTER reflectances $\left(\widehat{R}_{0.86, \mathrm{aA}}\right)$ in the VNIR. The gray diagonal line represents the $1: 1$ line. Overcast (partially cloudy) pixels are indicated in black (gray) color. Data are from observations on 13 May 2003 (C14). (b) Same as (a) but for $\widehat{R}_{2.1, \mathrm{M}}$ and $\widehat{R}_{2.1, \text { aA }}$ sampled in the SWIR. (c)-(d) Same as (a)-(b) but for observations on 10 June 2005 (C19).

\subsection{Case studies}

\subsubsection{Reflectance comparison for C14 and C19}

Figure 10a shows a comparison between atmospherically corrected VNIR reflectances sampled by MODIS $\left(\widehat{R}_{0.86, \mathrm{M}}\right)$ and co-located $\widehat{R}_{0.86 \text {,aA }}$ for the homogeneous scene C14. Only pixels containing liquid water clouds and both a successful MODIS and ASTER cloud property retrieval are included in the analysis. For the sake of display only, the ratio $f_{0.86, \mathrm{~L}}$ between the ASTER and MODIS LUT reflectances in the VNIR is calculated for the respective $\mathrm{C} 14$ geometry and $\left(\tau_{\mathrm{aA}}, r_{\mathrm{eff}, \mathrm{aA}}\right)$ pair and multiplied with $\widehat{R}_{0.86, \mathrm{aA}}$. This accounts for the theoretical differences in the respective SRF between both instruments (see Sect. 3.3). A strong positive correlation with $R=0.993$ is observed and all observations lie close to the $1: 1$ line. However, there seems to be a slight slope in the reflectance relation, as sampled $\widehat{R}_{0.86 \text {,aA }}$ appears to be slightly darker than $\widehat{R}_{0.86, \mathrm{M}}$ for brighter pixels. This is likely due to uncertainties in the calculation of $f_{0.86, \mathrm{~L}}$ (discussed in Sect. 5.4) and does not affect the cloud property retrieval. The correlation between $\widehat{R}_{2.1, \mathrm{M}}$ and $\widehat{R}_{2.1, \mathrm{aA}}$ sampled in the SWIR (and multiplied with the respective $f_{2.1, \mathrm{~L}}$ ) is shown in Fig. 10b. Similar to the VNIR observations, there is a high agreement with $R=0.936$. Again, there seems to be a slight slope in the reflectance relation, which is likely induced by uncertainties in the calculation of $f_{2.1, \mathrm{~L}}$.

Similar scatter plots for atmospherically corrected reflectances in the VNIR and SWIR sampled over the more inhomogeneous scene C19 are shown in Figs. 10c-d. The data set is sorted into overcast and partially cloudy pixels, respectively, determined from the subpixel cloud cover $C_{\text {sub }}$ based on ASTER cloudiness flags " 0 " and " 1 " at $15 \mathrm{~m}$ horizontal resolution. Overcast pixels exhibit $C_{\text {sub }}=1.0$, while partially cloudy pixels are characterized by $C_{\text {sub }}<1.0$. Even higher correlation coefficients of $R=0.997$ and $R=0.990$ are observed, respectively, and overall there is a good agreement between the MODIS and co-located ASTER observations. While reflectances from both instruments are mostly comparable, $\widehat{R}_{0.86, \mathrm{M}}\left(\widehat{R}_{2.1, \mathrm{M}}\right)$ are slightly larger than $\widehat{R}_{0.86, \mathrm{aA}}$ $\left(\widehat{R}_{2.1, \mathrm{aA}}\right)$ for brighter pixels.

For all overcast pixels sampled in $\mathrm{C} 14$ and $\mathrm{C} 19$, the remaining bias between $\widehat{R}_{0.86, \mathrm{aA}}$ and $\widehat{R}_{0.86, \mathrm{M}}$, after correcting for the theoretical difference due to their respective SRF, is about $3.5 \%$. Likewise, the remaining bias between $\widehat{R}_{2.1, \mathrm{aA}}$ and $\widehat{R}_{2.1, \mathrm{M}}$ is about $0.3 \%$. These values are in the range of the intercomparison results by Uprety et al. (2013), who reported radiometric bias uncertainties between the Suomi National Polar-Orbiting Partnership (Suomi NPP) VIIRS and MODIS in the range of $2-3 \%$ for the VNIR and SWIR 

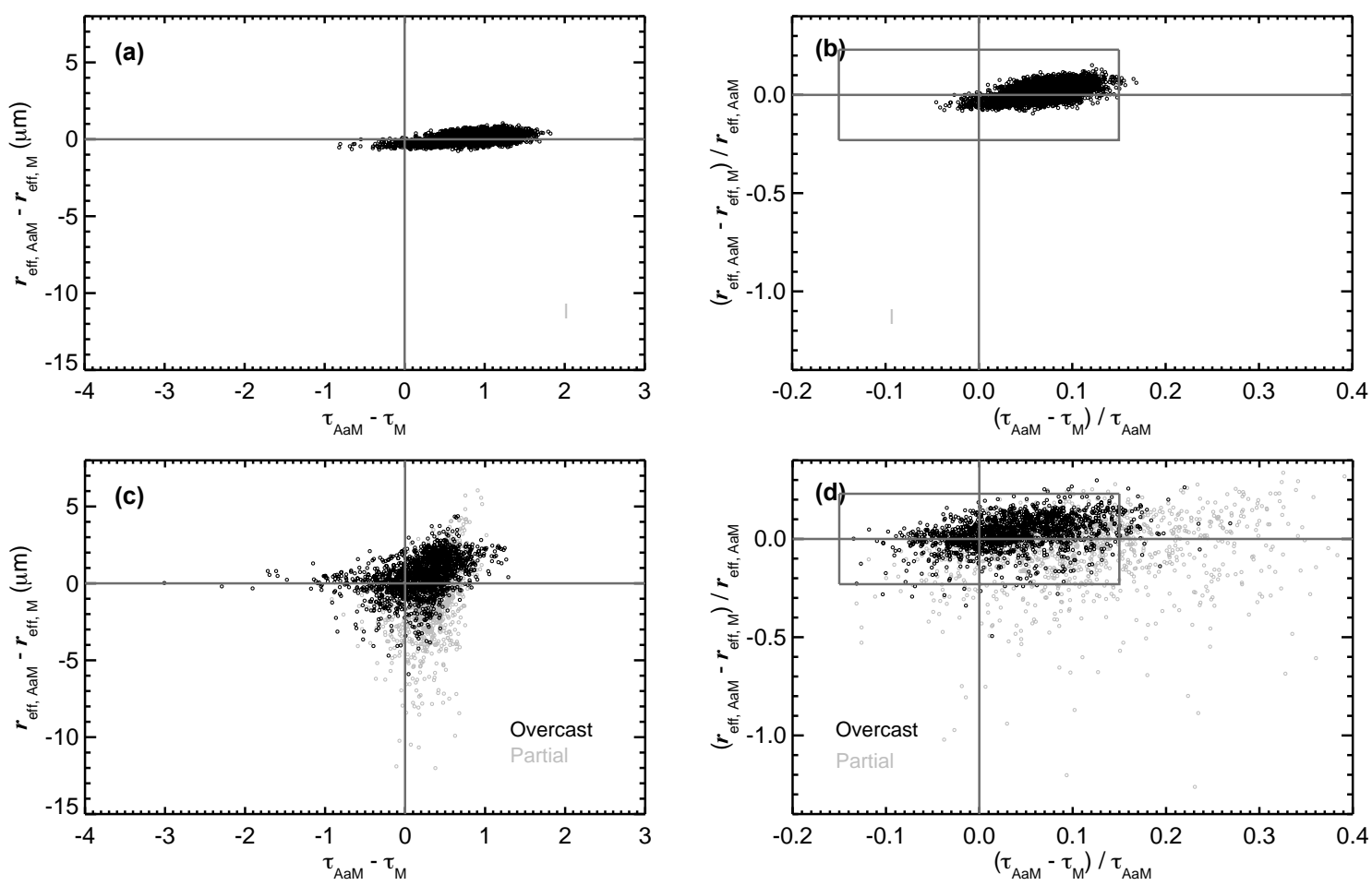

Figure 11. (a) Difference between effective droplet radius retrieved from co-located ASTER observations $\left(r_{\text {eff,aA }}\right)$ and the operational MODIS product $\left(r_{\text {eff,M }}\right)$ versus difference between cloud optical thickness retrieved from co-located ASTER observations $\left(\tau_{\mathrm{aA}}\right)$ and the operational MODIS product $\left(\tau_{\mathrm{M}}\right)$. Only data points where both ASTER and MODIS retrievals have a successful liquid water cloud retrieval are shown. Colors indicate samples over overcast (black) and partially cloudy pixels (gray). The gray horizontal and vertical lines indicate the points where no deviation between ASTER and MODIS retrievals occur. Data are from observations on 13 May 2003 (C14). (b) Same as (a) but normalized by $r_{\mathrm{eff}}$,aA and $\tau_{\mathrm{aA}}$, respectively. The gray box indicates the mean retrieval uncertainty for $r_{\mathrm{eff}}$,aA and $\tau_{\mathrm{aAA}}$, calculated by applying the absolute radiometric uncertainties of ASTER band $3 \mathrm{~N}$ and 5 reflectances. (c)-(d) Same as (a)-(b) but for observations on 10 June 2005 (C19).

bands. It is important to note that small differences in cloud top reflectances could result in possibly large differences in retrieved cloud properties.

\subsubsection{Retrieved cloud property comparison for C14 and C19}

There is a good agreement between the ASTER (at native resolution) and MODIS retrieval results shown in Figs. 7-8, with both instruments covering a similar value range and spatial distribution for $\tau_{\mathrm{A}}$ and $\tau_{\mathrm{M}}$, as well as $r_{\mathrm{eff}, \mathrm{A}}$ and $r_{\mathrm{eff}, \mathrm{M}}$. To gain a better understanding about the difference in retrieved cloud properties from aggregated ASTER reflectances and the respective MODIS C6 products, scatter plots of the difference $\tau_{\mathrm{aA}}-\tau_{\mathrm{M}}$ versus the difference $r_{\mathrm{eff}, \mathrm{aA}}-r_{\mathrm{eff}, \mathrm{M}}$ are shown in Fig. 11a for the homogeneous example cases C14. Samples over overcast pixels are illustrated by black circles, while gray circles indicate observations over partially cloudy pixels. Again, only pixels containing liquid water clouds and both a successful MODIS and ASTER retrieval are considered in the analysis.
Overall, there is a high agreement between the retrieved cloud properties from ASTER and MODIS, with minimum and maximum differences between $\tau_{\mathrm{aA}}$ and $\tau_{\mathrm{M}}$ of -0.85 and 1.82. Similarly, the observed minimum and maximum differences between $r_{\text {eff, aA }}$ and $r_{\mathrm{eff}, \mathrm{M}}$ are -0.70 and $1.09 \mu \mathrm{m}$. For $\mathrm{C} 14$, all pixels are characterized by $C_{\mathrm{sub}}=1.0$. The median difference in retrieved optical thickness (effective droplet radius) is $0.87(0.13 \mu \mathrm{m})$ with an IQR of $0.43(0.48 \mu \mathrm{m})$. For $\mathrm{C} 14$ there seems to be a slight bias in retrieved $\tau_{\mathrm{A}}$ of about 0.7 .

To relate these differences to the ASTER retrieval uncertainties derived in Sect. 2.3, the differences $\tau_{\mathrm{aA}}-\tau_{\mathrm{M}}$ and $r_{\text {eff,aA }}-r_{\text {eff, }}$ are normalized by $\tau_{\mathrm{aA}}$ and $r_{\text {eff, aA }}$, respectively. The results for $\mathrm{C} 14$ are shown in Fig. 11b, where the gray box indicates the retrieval uncertainty for both cloud variables due to radiometric uncertainties only (see the discussion in Sect. 3.2.2). It is obvious that the differences in retrieved optical thickness and effective droplet radius between ASTER and MODIS are well within the retrieval uncertainties of ASTER. The best agreement between the two sensors is achieved for bright cloudy pixels where $\tau_{\mathrm{M}}, \tau_{\mathrm{aA}} \geq 14$. Here, differences in retrieved optical thickness are in the 
range of $5 \%$, while differences in retrieved effective droplet radius are $\pm 10 \%$. With lower $R_{\mathrm{aA}}$ the retrieval differences, as well as the bias in $\tau_{\mathrm{aA}}$, increase.

The comparison of retrieved cloud properties for the more inhomogeneous example scene C19 is shown in Fig. 11c-d. Differences between $\tau_{\mathrm{aA}}$ and $\tau_{\mathrm{M}}$ range from -3.11 to +1.26 (with a median difference of 0.30 and an IQR of 0.55 ), while overcast pixels exhibit differences between $r_{\text {eff,aA }}$ and $r_{\text {eff,M }}$ in the range of -6.16 to $4.57 \mu \mathrm{m}$ (with a median difference of $0.53 \mu \mathrm{m}$ and an IQR of $1.64 \mu \mathrm{m}$ ). The largest differences in retrieved effective droplet radius between ASTER and MODIS are observed for partially cloudy pixels, where $\tau_{\mathrm{M}}$ and $\tau_{\mathrm{aA}}$ are low. Here, the difference $r_{\mathrm{eff}, \mathrm{aA}}-r_{\mathrm{eff}, \mathrm{M}}$ can be as large as $-12.36 \mu \mathrm{m}$. All these pixels are characterized by low $\tau_{\mathrm{M}}, \tau_{\mathrm{aA}}$ and the subpixel cloud cover $C_{\mathrm{sub}}$, derived from the original $15 \mathrm{~m}$ ASTER resolution for cloudiness flags " 0 " and "1" (i.e., "confidently" and "probably cloudy" pixels), can be as low as 0.730 . This implies that the retrieval is contaminated by low ocean surface reflectance observations. While there seemed to be a positive $\widehat{R}_{0.86, \mathrm{M}}$ and $\widehat{R}_{2.1, \mathrm{M}}$ bias for large reflectances, the optical thickness and effective droplet radius differences show no such bias (i.e., they are centered around $\tau_{\mathrm{aA}}-\tau_{\mathrm{M}}=0$ and $r_{\mathrm{eff}, \mathrm{aA}}-r_{\mathrm{eff}, \mathrm{M}}=0 \mu \mathrm{m}$, respectively).

Normalizing the retrieval differences with $\tau_{\mathrm{aA}}$ and $r_{\text {eff,aA }}$ illustrates that again almost all observations are within the retrieval uncertainties of the ASTER instrument. However, partially cloudy pixels yield differences between $\tau_{\mathrm{aA}}$ and $\tau_{\mathrm{M}}$ of up to $35 \%$ and overestimation in $r_{\mathrm{eff}, \mathrm{M}}$ (compared to $r_{\text {eff,aA }}$ ) of up to $130 \%$. Similar to C14, the best agreements between ASTER and MODIS cloud variables are achieved for bright pixels with high $\widehat{R}_{0.86, \text { aA }}$ and $\widehat{R}_{0.86, \mathrm{M}}$ (and subsequently high $\tau_{\mathrm{aA}}$ and $\tau_{\mathrm{M}}$ ). Here, the differences between both sensors are about $\pm 10 \%$ for both the optical thickness and effective droplet radius. With decreasing $\tau_{\mathrm{aA}}$ and $\tau_{\mathrm{M}}$ the differences increase up to the retrieval uncertainty of ASTER.

Overall, the correlation coefficients between $\tau_{\mathrm{aA}}$ and $\tau_{\mathrm{M}}$ are $R=0.992$ and $R=0.995$ for $\mathrm{C} 14$ and C19, respectively. The correlation of $r_{\mathrm{eff}, \mathrm{M}}$ and $r_{\mathrm{eff}, \text { aA }}$ yields $R=0.872$ for $\mathrm{C} 14$ and $R=0.739$ for C19. Limiting the analysis to observations with overcast pixels increases the correlation coefficient for the effective droplet radius comparison to $R=0.889$ for C19.

\subsection{Statistical comparison for $48 \mathrm{MBL}$ cloud scenes}

\subsubsection{Cloud mask comparison}

Co-located ASTER reflectances are used to get a cloud mask value for each pixel of the $48 \mathrm{MBL}$ cases, assigning the respective cloud mask flag according to the discussion in Sect. 3.1. To compare the domain-averaged cloud cover from ASTER observations $\left(C_{\mathrm{aA}}\right.$, derived from the aggregated ASTER radiances) with the operational MODIS results from the MOD35 data containers $\left(C_{\mathrm{M}}\right)$, the fraction of pixels with

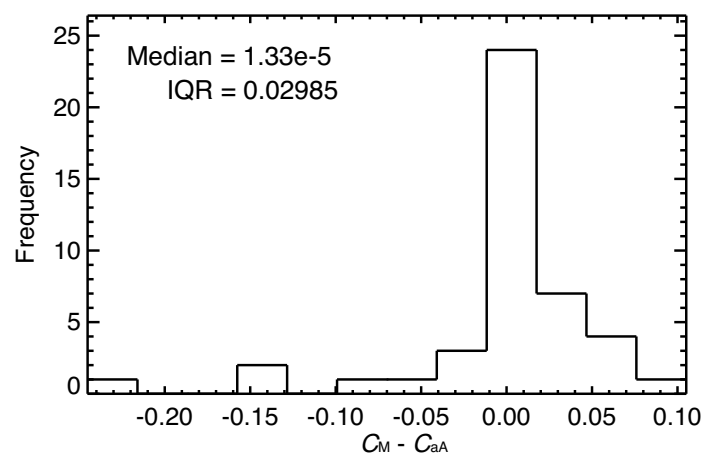

Figure 12. Difference in domain-averaged cloud cover based on the operational MODIS cloud mask $\left(C_{\mathrm{M}}\right)$ and co-located ASTER observations $\left(C_{\mathrm{aA}}\right)$. Values for the median and interquartile range (IQR) are given.

a cloudiness flag value of " 0 " or " 1 " is calculated (i.e., the fraction of "confidently" and "probably cloudy" pixels).

Figure 12 shows a frequency distribution of the difference between the domain-averaged cloud covers from MODIS and co-located ASTER measurements. An agreement between $C_{\mathrm{M}}$ and $C_{\mathrm{aA}}$ of \pm 0.04 is observed for 34 of the 48 analyzed MBL scenes (i.e., $73.9 \%$ ), while $89.1 \%$ of cases exhibit an agreement in scene cloud cover of \pm 0.1 . Cases where the absolute difference between $C_{\mathrm{M}}$ and $C_{\mathrm{aA}}$ is larger than 0.1 are characterized by $C_{\mathrm{M}}=0.11-0.87$ and include a substantial number of pixels characterized by MODIS cloudiness flags " 2 " (i.e., "probably clear" pixels). For these scenes, transitioning observations with cloudiness flags " 2 " to cloudiness flags " 1 " (i.e., assuming these pixels are "probably cloudy" instead of "probably clear" pixels) improves the agreement between the MODIS and ASTER domainaveraged cloud covers, which implies that the biggest discrepancies between $C_{\mathrm{M}}$ and $C_{\mathrm{aA}}$ are caused by pixels with very thin clouds. Overall the median difference between $C_{\mathrm{M}}$ and $C_{\mathrm{aA}}$ is basically 0 with an IQR of 0.03 .

On the pixel level, $91.4 \%$ of the cloudy pixels, as identified by the MODIS cloudiness flags " 0 " and " 1 ", are also flagged by the ASTER cloud masking scheme. Similarly, $94.0 \%$ of clear MODIS pixels are characterized as clear ASTER pixels. Of all cloudy MODIS pixels $7.1 \%$ are missed by the ASTER algorithm and are characterized by a failed cloud property retrieval, while $1.4 \%$ exhibit $0>\tau_{\mathrm{aA}}<5$.

\subsubsection{Reflectance comparison for $48 \mathrm{MBL}$ cloud scenes}

Figure 13a shows probability density functions (PDFs) of $\widehat{R}_{0.86, \mathrm{M}}$ (green lines) and $\widehat{R}_{0.86, \mathrm{aA}}$ (black lines) sampled in the VNIR. Data are from all 48 MBL scenes, but only overcast pixels are considered. As described in Sect. 5.2.1, overcast pixels are characterized by a subpixel cloud cover $C_{\text {sub }}=1.0$. Although these data points theoretically include the operational MODIS PCL observations, practically no PCL pixels remain with the $C_{\text {sub }}=1.0$ constraint. Only pix- 

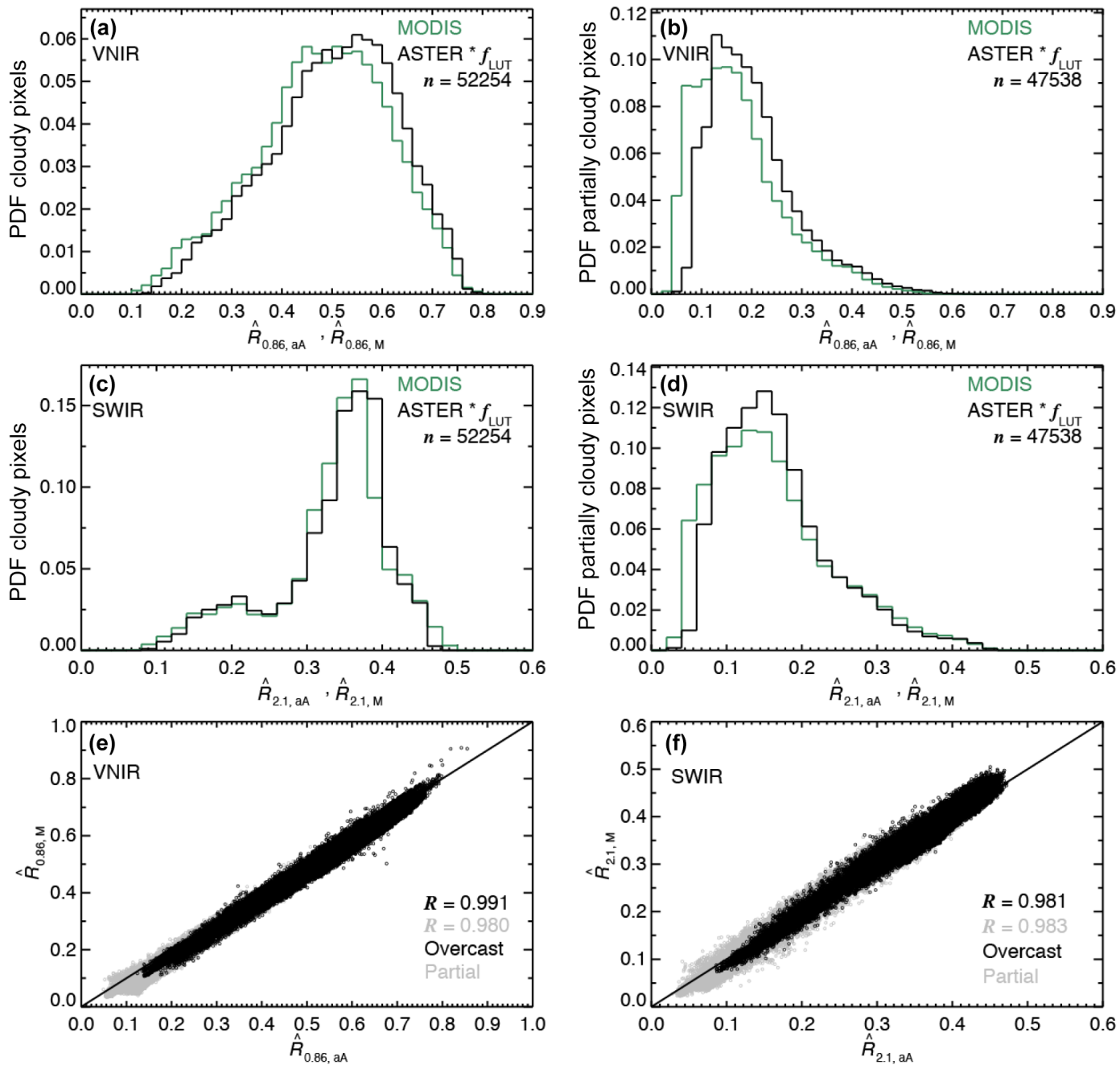

Figure 13. (a) PDFs of atmospherically corrected MODIS VNIR reflectances $\left(\widehat{R}_{0.86, \mathrm{M}}\right)$ and co-located, atmospherically corrected ASTER VNIR reflectances $\left(\widehat{R}_{0.86, \mathrm{aA}}\right)$, multiplied by the derived ratio between ASTER and MODIS LUT reflectances $\left(f_{0.86, \mathrm{~L}}\right.$, see Sect. 2.3$)$. Only overcast pixels, containing both a successful MODIS and ASTER liquid water cloud retrieval, from the 48 MBL cases are considered in the calculation of the PDFs. The number of samples $(n)$ is given. (b) Same as (a) but for partially cloudy pixels. (c)-(d) Same as (a)-(b) but for $\widehat{R}_{2.1, \mathrm{M}}$ and $\widehat{R}_{2.1, \text { aA }}$ sampled in the SWIR. (e) Scatter plot of $\widehat{R}_{0.86, \mathrm{M}}$ and $\widehat{R}_{0.86, \text { aA }}$ (multiplied by $f_{2.1, \mathrm{~L}}$ ) in the VNIR. Overcast (partially cloudy) pixels are indicated in black (gray) color. The diagonal line represents the $1: 1$ line. (f) Same as (e) but for reflectances in the SWIR

els containing liquid water clouds and both a successful MODIS and ASTER cloud property retrieval are considered. This yields $n=52254$ observations for the $48 \mathrm{MBL}$ cases. For the sake of display only, the ratio $f_{0.86, \mathrm{~L}}$ between the ASTER and MODIS LUT reflectances in the VNIR is derived for each observation and multiplied with $\widehat{R}_{0.86, \text { aA }}$. Both $\widehat{R}_{0.86, \mathrm{M}}$ and $\widehat{R}_{0.86 \text {,aA }}$ show a similar distribution with most observations of $\widehat{R}_{0.86, \mathrm{M}}, \widehat{R}_{0.86, \mathrm{aA}}=0.107-$ 0.908 . ASTER observations are slightly higher with mean $\widehat{R}_{0.86, \mathrm{aA}}=0.501$ compared to mean $\widehat{R}_{0.86, \mathrm{M}}=0.479$.

PDFs of $\widehat{R}_{0.86, \mathrm{M}}$ and $\widehat{R}_{0.86 \text {,aA }}$ for partially cloudy pixels are illustrated in Fig. 13b. Similar to the definition of overcast pixels, partially cloudy pixels are characterized by subpixel cloud covers $C_{\text {sub }}<1.0$ and include the operational MODIS
PCL observations. The number of partially cloudy pixels is slightly smaller than the number of overcast pixels with $n=$ 47 538. Both ASTER and MODIS reflectances show similar ranges of $\widehat{R}_{0.86, \mathrm{M}}, \widehat{R}_{0.86, \mathrm{aA}}=0.033-0.631$. Mean $\widehat{R}_{0.86, \mathrm{aA}}=$ 0.204 is again slightly higher than mean $\widehat{R}_{0.86, \mathrm{M}}=0.176$.

Figure $13 \mathrm{c}-\mathrm{d}$ show PDFs of SWIR reflectances $\widehat{R}_{2.1, \mathrm{M}}$ and $\widehat{R}_{2.1 \text {, aA }}$ for overcast and partially cloudy pixels, respectively. As for the VNIR observations, the SWIR $\widehat{R}_{2.1, \mathrm{aA}}$ are multiplied with the respective ratio $f_{2.1, \mathrm{~L}}$. Both $\widehat{R}_{2.1, \mathrm{M}}$ and $\widehat{R}_{2.1 \text { aA }}$ for overcast pixels are characterized by a bimodal distribution, with values of $\widehat{R}_{2,1, \mathrm{M}}, \widehat{R}_{2,1, \mathrm{aA}}=0.074$ 0.505 and maximum values around $\widehat{R}_{2.1, \mathrm{M}}, \widehat{R}_{2.1, \mathrm{aA}}=0.2$ and $\widehat{R}_{2.1, \mathrm{M}}, \widehat{R}_{2.1, \mathrm{aA}}=0.35$. Mean $\widehat{R}_{2.1 \mathrm{aA}}=0.333$, which compares well to the mean $\widehat{R}_{2.1, \mathrm{M}}=0.331$. Observed $\widehat{R}_{2.1, \mathrm{M}}$ 
and $\widehat{R}_{2.1}$ aA in the SWIR for partially cloudy pixels range within $\widehat{R}_{2.1, \mathrm{M}}, \widehat{R}_{2.1, \mathrm{aA}}=0.023-0.474$. Mean observations agree well with mean $\widehat{R}_{2.1, \mathrm{aA}}=0.162$ compared to mean $\widehat{R}_{2.1, \mathrm{M}}=0.168$.

The good agreement between the ASTER and MODIS reflectances is also illustrated in the scatter plots in Fig. 13e$\mathrm{f}$, for VNIR and SWIR reflectances, respectively. Here, observations over overcast (partially cloudy) pixels are shown with black (gray) dots, while the diagonal black line represents the $1: 1$ line. Derived $\widehat{R}_{0.86, \mathrm{M}}$ and $\widehat{R}_{0.86, \mathrm{aA}}$ in the VNIR, as well as $\widehat{R}_{2.1, \mathrm{M}}$ and $\widehat{R}_{2.1, \mathrm{aA}}$ in the SWIR, lie close to the $1: 1$ line with high Pearson's product-moment correlation coefficients of $R=0.996$ ( $R=0.991$ for overcast pixels and $R=0.980$ for partially cloudy pixels) and $R=0.992$ ( $R=0.981$ for overcast pixels and $R=0.983$ for partially cloudy pixels), respectively. However, the slight slopes observed for the example cases C14 and C19 are also apparent for the complete data set. For the VNIR and SWIR reflectances the linear fit functions yield slope values of $\alpha_{0.86}=$ 1.020 and $\alpha_{2.1}=1.036$, respectively, with offset values of $\beta_{0.86}=-0.033$ (for VNIR reflectances) and $\beta_{2.1}=-0.014$ (for SWIR reflectances). This confirms the results found for the two example cases, where ASTER reflectances are slightly higher than the MODIS observations at the lower end, and vice versa for higher reflectances.

\subsubsection{Retrieved cloud property comparison for 48 MBL cloud scenes}

PDFs of $\tau_{\mathrm{M}}$ (green lines) and $\tau_{\mathrm{aA}}$ (black lines) from observations over all $48 \mathrm{MBL}$ scenes are shown in Fig. 14a. Similar to the reflectance comparison earlier, only overcast pixels containing liquid water clouds and a successful MODIS and ASTER cloud property retrieval are considered in the analysis. The distribution of $\tau_{\mathrm{aA}}$ agrees well with the MODIS product and, consistent with the slightly higher VNIR reflectances, $\tau_{\mathrm{aA}}$ are slightly higher than $\tau_{\mathrm{M}}$ with mean $\tau_{\mathrm{aA}}=$ 12.29 , compared to mean $\tau_{\mathrm{M}}=11.42$. Although observations can reach values as high as $\tau_{\mathrm{M}}=90.17$, over $99.9 \%$ of pixels exhibit $1.37 \geq \tau_{\mathrm{M}}, \tau_{\mathrm{aA}} \leq 30.00$. Restricting the analysis to partially cloudy pixels only, shown in Fig. 14b, yields a narrower distribution with retrieved $\tau_{\mathrm{M}}, \tau_{\mathrm{aA}}=0.17-24.92$. Here, the center of each distribution is significantly reduced from the overcast data set with mean $\tau_{\mathrm{aA}}=3.62$ and mean $\tau_{\mathrm{M}}=3.08$.

The distributions of $r_{\text {eff, }}$ and co-located $r_{\text {eff, aA }}$ for overcast pixels are shown in Fig. 14c, illustrating a good agreement between both instruments. While the retrievals can be as low as $r_{\text {eff, },}, r_{\text {eff,aA }}=4.76 \mu \mathrm{m}$, the upper limit for both the ASTER and MODIS retrieval is a fixed value of $r_{\text {eff,M }}, r_{\text {eff, aA }}=30.00 \mu \mathrm{m}$. For larger droplets the LUTs converge and the retrieval results become unreliable. For all $48 \mathrm{MBL}$ scenes the mean $r_{\mathrm{eff}, \mathrm{aA}}=10.07 \mu \mathrm{m}$, which compares well with the mean observed $r_{\text {eff, }}=9.93 \mu \mathrm{m}$. Figure $14 \mathrm{~d}$ shows the PDFs of $r_{\text {eff, } \mathrm{M}}$ and $r_{\mathrm{eff}, \mathrm{aA}}$ for partially cloudy pixels. Although there seem to be more observations in the range $10.00 \mu \mathrm{m} \leq r_{\text {eff, }}, r_{\text {eff, aA }} \leq 20.00 \mu \mathrm{m}$, the mean values are only slightly increased to mean $r_{\text {eff, aA }}=11.48 \mu \mathrm{m}$ and mean $r_{\mathrm{eff}, \mathrm{M}}=10.60 \mu \mathrm{m}$.

Figure $14 \mathrm{e}$ illustrates a scatter plot of $\tau_{\mathrm{M}}$ and $\tau_{\mathrm{aA}}$ sampled over all available overcast (black dots) and partially cloudy (gray dots) pixels. There is a good agreement between the results from both instruments, with most observations close to the $1: 1$ line and a correlation coefficient of $R=0.992$ ( $R=0.979$ for overcast pixels and $R=0.968$ for partially cloudy pixels). The concentration of data points around $0<\tau_{\mathrm{aA}}, \tau_{\mathrm{M}}<5$ illustrates that partially cloudy pixels are characterized by very low optical thicknesses. A scatter plot of $r_{\text {eff, } \mathrm{M}}$ and $r_{\text {eff,aA }}$ is shown in Fig. 14f. While there is a good agreement for overcast pixels, illustrated by $R=0.972$, there are visible deviations for partially cloudy pixels $(R=0.739)$. As shown in Fig. $14 \mathrm{e}$, most partially cloudy pixels exhibit $\tau_{\mathrm{M}}, \tau_{\mathrm{aA}}<5$. In this part of the LUT the retrieval sensitivity is very low and even small uncertainties in $R_{\mathrm{M}}$ and $R_{\mathrm{aA}}$ yield large retrieval uncertainties for both $r_{\text {eff, }}$ and $r_{\text {eff,aA }}$. The fact that the PDFs of $r_{\text {eff,M }}$ and $r_{\text {eff,aA }}$ agree well shows that there is no preferred sign in the deviations (i.e., there is no overall overestimation or underestimation by one instrument). This implies that the relation between both retrieval products mostly resembles noise, indicating that retrieval uncertainties are the cause for the discrepancies. Including observations from partially cloudy pixels reduces the correlation coefficient for all $r_{\mathrm{eff}, \mathrm{M}}$ and $r_{\text {eff,aA }}$ to $R=0.851$. The slight slopes in the reflectance relations yields slight slopes in the cloud property relations. For overcast pixels the derived slope values are $\alpha_{\tau}=1.016$ (for the cloud optical thickness) and $\alpha_{\mathrm{r}}=1.091$ (for the effective droplet radius), while the offset values are $\beta_{\tau}=-1.069$ and $\beta_{\mathrm{r}}=-1.061$. This implies a slight underestimation (overestimation) of the ASTER retrievals on the low (high) end of the respective value ranges.

\subsection{Uncertainty contributions}

The analysis in Sects. 5.2.1-5.3.3 reveals a high agreement between the operational MODIS cloud retrieval products and the co-located ASTER results. This can be attributed to the use of the MODIS C6 retrieval algorithms and radiative transfer codes. Still, remaining uncertainties lead to the small differences in the cloud variable comparison. Besides the radiometric uncertainties of each instrument, a number of factors impact the comparison between the MODIS and ASTER results.

Differences in the center wavelengths and SRF between the ASTER and MODIS bands, while theoretically accounted for in the applied radiative transfer codes, yield a remaining uncertainty not only in the reflectance comparison but also in the retrieved cloud top, optical, and microphysical properties. While the transmittance tables used in the retrieval algorithm of cloud top properties are calculated for 

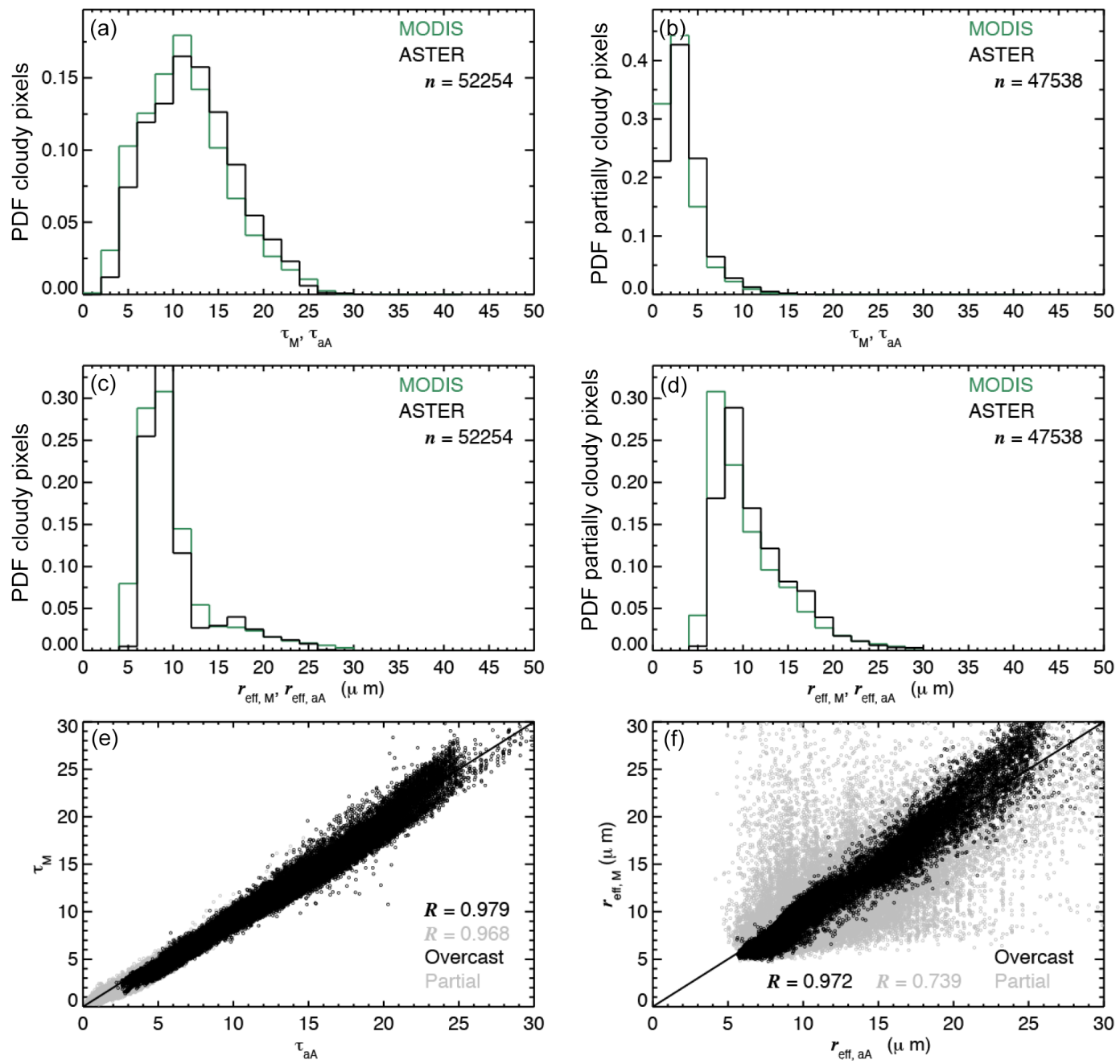

Figure 14. (a) PDFs of cloud optical thickness retrieved from MODIS reflectances $\left(\tau_{\mathrm{M}}\right)$ and co-located ASTER reflectances $\left(\tau_{\mathrm{aA}}\right)$. Only overcast pixels, containing both a successful MODIS and ASTER liquid water cloud retrieval, from the 48 MBL cases are considered in the calculation of the PDFs. The number of samples $(n)$ is given. (b) Same as (a) but for partially cloudy pixels. (c)-(d) Same as (a)-(b) but for the effective droplet radius retrieved from MODIS reflectances $\left(r_{\mathrm{eff}, \mathrm{M}}\right)$ and co-located ASTER reflectances $\left(r_{\mathrm{eff}, \mathrm{AA}}\right)$. (e) Scatter plot of $\tau_{\mathrm{M}}$ and $\tau_{\mathrm{aA}}$. Overcast (partially cloudy) pixels are indicated in black (gray) color. The diagonal line represents the 1:1 line. (f) Same as (e) but for $r_{\text {eff,M }}$ and $r_{\text {eff,aA }}$.

the full ASTER SRFs, the operational MODIS IR window retrieval and optimal estimation method are applied to the ASTER IR observations without any threshold adjustments. Spectral differences also impact the atmospheric correction scheme. Because the ASTER VNIR band covers absorption features of atmospheric oxygen $\left(\mathrm{O}_{2} \mathrm{~A}\right.$ band $)$ and water vapor, it is more sensitive to the atmospheric correction scheme than the respective MODIS VNIR band. The sensitivity has been derived by means of a susceptibility analysis, similar to the method described in Werner et al. (2014). The susceptibility $S$ is defined as the relative change of the ratio of uncorrected to corrected reflectance $\left(\widehat{R}_{0.86, \mathrm{aA}} / R_{0.86, \mathrm{aA}}\right)$ with a change in cloud top height $z_{\text {top }}$, which for the collocated ASTER VNIR data can be written as

$$
\begin{aligned}
S & =\frac{\mathrm{d}\left(\widehat{R}_{0.86, \mathrm{aA}} / R_{0.86, \mathrm{aA}}\right)}{\mathrm{d} z_{\text {top }}} \cdot \frac{z_{\text {top }}}{\left(\widehat{R}_{0.86, \mathrm{aA}} / R_{0.86, \mathrm{aA}}\right)} \\
& =\frac{\mathrm{d} \ln \left(\widehat{R}_{0.86, \mathrm{aA}} / R_{0.86, \mathrm{aA}}\right)}{\mathrm{d} \ln z_{\text {top }}} .
\end{aligned}
$$

Deriving $S$ for all 48 MBL cloud scenes yields similar values of -0.025 and -0.024 for the ASTER and MODIS SWIR bands, respectively, indicating a similar sensitivity towards the atmospheric correction for both instruments. Conversely, $S$ in the VNIR bands is -0.021 (ASTER) and -0.006 (MODIS), indicating that measurements in the ASTER VNIR band are significantly more sensitive to the atmospheric correction scheme than the respective MODIS 
measurements. This also implies that sampled reflectances in the ASTER VNIR band are more susceptible to uncertainties in the atmospheric correction scheme. However, the research-level retrieval algorithm presented in this paper employs the same ancillary data sets, as well as the extensively documented and tested atmospheric correction algorithm implemented in the operational MODIS C6 code. The good agreement between $\widehat{R}_{0.86, a A}$ and $\widehat{R}_{0.86, \mathrm{M}}$, shown in Fig. 13e-f, can be attributed to the reliability of this scheme. Since the land surface albedo product is created for MODIS bands 1-7 and there is no specific surface albedo product for ASTER, the SRF differences between ASTER and MODIS bands also induce uncertainties in the derived spectral surface albedo values. This is acknowledged by an increase in surface albedo uncertainty from 15 to $30 \%$ in the pixellevel uncertainty calculations. However, since the focus of this study is on MBL clouds sampled over ocean, this effect is mitigated by the use of ocean surface reflectances derived from the Cox-Munk model generated using the precise ASTER SRF. For the reflectance comparison, the ratios $f_{0.86, \mathrm{~L}}$ and $f_{2.1, \mathrm{~L}}$ theoretically provide the means to compare $\widehat{R}_{0.86, \mathrm{M}}$ and $\widehat{R}_{0.86, \mathrm{aA}}$, as well as $\widehat{R}_{2.1, \mathrm{M}}$ and $\widehat{R}_{2.1, \mathrm{aA}}$. However, both are derived by means of radiative transfer simulations and are thus impacted by the involved assumptions (e.g., the ocean surface albedo, which might be different to the actually observed albedo). As mentioned in Sect. 3.3, derived $\tau_{\mathrm{M}}$ and $\tau_{\mathrm{aA}}$ are scaled to MODIS band 1 and ASTER band 2, respectively (both centered around $0.65 \mu \mathrm{m}$ ). This means that the comparison between $\tau_{\mathrm{M}}$ and $\tau_{\mathrm{aA}}$ is also influenced by the different center wavelengths and SRF of this band. These differences are in the range of the applied VNIR band (centered around $0.86 \mu \mathrm{m})$. The band differences also result in different vertical weighting functions (Platnick, 2000), which describe the vertical photon transport within the cloud and impact the retrieval of $r_{\mathrm{eff}, \mathrm{M}}$ and $r_{\mathrm{eff}, \mathrm{aA}}$.

The aggregation of digital ASTER counts $d_{\mathrm{A}}(\Delta \lambda)$ within a $1000 \times 1000 \mathrm{~m}$ MODIS pixel is described in Sect. 5.1 and Fig. 9. The co-location of ASTER and MODIS samples benefits from the small horizontal resolution of the ASTER measurements, the position of both instruments aboard Terra and an almost identical alignment of the respective pixels (i.e., pixel edges are almost parallel). Still, small colocation uncertainties remain. Here, a significant contribution comes from the full aggregation of $d_{\mathrm{A}}(\Delta \lambda)$ of ASTER pixels that are only partially within a MODIS pixel (right at the MODIS pixel edges). The resulting uncertainties have been derived for a number of example MODIS pixels, where the $d_{\mathrm{A}}(\Delta \lambda)$ values of partially included ASTER pixels have been weighted according to the respective area within the MODIS pixel. These computationally expensive calculations reveal an uncertainty in the aggregated digital counts of $<0.05$. However, the effect of this uncertainty is mitigated by the fact that the ASTER signal is stored as 8 bit unsigned integer values. Thus, aggregated ASTER counts are rounded to full integer values and cover a possible range of $0-255$. The resulting rounding error yields uncertainties in the derived reflectances $R_{0.86, \mathrm{aA}}$ and $R_{2.1 \text {, aA }}$, which get higher when the signal gets darker. For the 48 scenes presented in this paper, the maximum reflectance uncertainty introduced by this rounding error is $5 \%$, associated with cloudy pixels characterized by $R_{0.86, \mathrm{aA}} \approx 0.03$. Moreover, uncertainties arise due to the MODIS point spread function (PSF), which characterizes the signal distribution within and outside a MODIS pixel (Huang et al., 2002). While $d_{\mathrm{A}}(\Delta \lambda)$ from all ASTER samples contributes equally to the aggregated signal, the MODIS PSF implies that the largest contribution in a MODIS signal comes from the center of the pixel, while there is also a noticeable influence from surrounding pixels.

Differences in $\theta_{\mathrm{s}}$ between ASTER and MODIS, which are $\Delta \theta_{\mathrm{s}}<0.8^{\circ}$ and $\Delta \theta_{\mathrm{s}}<0.2^{\circ}$ for the two example cases $\mathrm{C} 14$ and $\mathrm{C} 19$, respectively, can result in significant differences in the retrieved cloud variables. Especially around the cloudbow and glory region uncertainties in the scattering angle can have a large impact on the sampled reflectances from both sensors. Moreover, for clouds with highly heterogeneous cloud tops small differences in $\theta_{\mathrm{S}}$ imply that different parts of the cloud are sampled by each instrument.

Electronic crosstalk, which causes visible striping in the MODIS cloud property retrievals shown in Fig. $8 \mathrm{~b}$ and d, induces additional uncertainties when comparing the operational MODIS and co-located ASTER results.

The pixel-level uncertainty estimates for retrieved cloud products based on ASTER observations are comprised of uncertainties in surface albedo, radiometric calibration, the applied models, and the amount of above-cloud precipitable water. This closely follows the MODIS Collection 6 approach and yields similar retrieval uncertainties for both the ASTER and MODIS results. However, while for ASTER the calibration and model uncertainties are assumed to be a constant value of $5 \%$, uncertainties in the operational MODIS C6 cloud products include spectral, scene-dependent Level 1B uncertainty indices (Sun et al., 2012).

The ASTER cloud property retrieval at $15 \mathrm{~m}$ horizontal resolution requires that SWIR reflectances are scaled up to match the resolution of the VNIR band $3 \mathrm{~N}$. To estimate the retrieval uncertainties it is assumed that the variability of four SWIR reflectance samples at $30 \mathrm{~m}$ resolution within a $60 \times 60 \mathrm{~m}$ pixel is similar to the variability of four SWIR reflectance samples at $15 \mathrm{~m}$ resolution within a $30 \times 30 \mathrm{~m}$ pixel. For the data set presented in this study statistics were derived about the difference $\Delta R_{2.1,30-60}$ between actually observed SWIR reflectances at $30 \mathrm{~m}$ resolution and replicated values from the $60 \mathrm{~m}$ samples. The median $\Delta R_{2.1,30-60}$ from over 4.3 million pixels is naturally $0 \%$. The 10 th and 90 th, as well as the 25th and 75th, percentiles of $\Delta R_{2.1,30-60}$ are \pm 3.1 and $\pm 1.3 \%$, respectively. This means that for most observations the uncertainties in retrieved $\tau_{\mathrm{A}}$ and $r_{\mathrm{eff}, \mathrm{A}}$ due to the replication of SWIR reflectances at the highest ASTER resolution are estimated to be less than \pm 0.5 and $\pm 0.7 \mu \mathrm{m}$, respectively. However, since the comparison between MODIS 
and co-located ASTER results is done with aggregated digital counts and not the $15 \mathrm{~m}$ data, it is not affected by the replication of ASTER SWIR observations.

\section{Summary and conclusions}

This study presents MODIS-like cloud property retrievals of MBL cloud optical and microphysical properties from high spatial resolution observations of the ASTER instrument aboard Terra. The ASTER retrievals of $\tau_{\mathrm{A}}$ and $r_{\mathrm{eff}, \mathrm{A}}$, with a horizontal resolution as low as $15 \mathrm{~m}$, are enabled by a research-level retrieval algorithm, which utilizes the operational MODIS C6 algorithm core.

The first objective of this paper is to document the retrieval scheme. The retrieval is based on the bispectral retrieval approach with precalculated LUTs and sampled reflectances at ASTER bands $3 \mathrm{~N}$ (centered around $\lambda=0.810 \mu \mathrm{m}$ in the VNIR) and 5 (centered around $\lambda=2.165 \mu \mathrm{m}$ in the SWIR). Because the central wavelengths and spectral response functions of the ASTER bands differ from the respective MODIS bands, ASTER-specific LUTs are applied in the cloud property retrieval. Depending on the scene geometry the ASTER VNIR band can appear slightly brighter or darker than the respective MODIS band, with differences in the range of $\pm 2-$ $3 \%$. Simulated SWIR reflectances of ASTER are about $10 \%$ larger compared to the MODIS LUT. Since ASTER also lacks certain bands necessary for the MODIS cloud masking scheme, a new algorithm is introduced. It is based on five cloudiness thresholds and tested with about 210 ASTER MBL scenes ranging from homogeneous altocumulus to heterogeneous broken cloud fields. This data set also includes the cases presented in Zhao and Di Girolamo (2006), where cloud amount is determined by an individual, singleband threshold for each scene. Comparisons between derived scene cloud covers from this single-band threshold and the new cloud masking algorithm show a high agreement with a median difference of about $0.4 \%$. It is shown that pixels containing very thin clouds are potentially missed by the algorithm. However, only $0.03 \%$ of these pixels are characterized by $\tau_{\mathrm{A}} \geq 5$. The LUT collapses fast for $\tau_{\mathrm{A}}<5$, which significantly reduces the retrieval sensitivity and increases the uncertainties in the derived $\tau_{\mathrm{A}}$ and $r_{\text {eff, A. Examples of }}$ high-resolution ASTER retrievals are presented for two MBL scenes with different degrees of horizontal cloud heterogeneity. These cases demonstrate that the ASTER observations can resolve small-scale, highly heterogeneous cloud structures, which are significantly smoothed by the MODIS measurements.

The second objective of this study is to compare co-located ASTER retrievals to the operational MODIS C6 results. The data set is provided by $48 \mathrm{MBL}$ scenes sampled off the coast of California. To match the MODIS sampling geometry, the digital ASTER counts at the original 15-90 m horizontal resolution are aggregated within the respective MODIS pixels.
The ASTER retrieval algorithm subsequently provides colocated ASTER results of $\widehat{R}_{0.86, \mathrm{aA}}, \widehat{R}_{2.1, \mathrm{aA}}, \tau_{\mathrm{aA}}$, and $r_{\mathrm{eff}, \mathrm{aA}}$. Moreover, the ASTER cloud mask at $15 \mathrm{~m}$ horizontal resolution yields a subpixel cloud cover for each aggregated pixel, which is used to discriminate between overcast and partially cloudy pixels. The data set amounts to 52254 overcast and 47538 partially cloudy pixels, where both ASTER and MODIS contain successful liquid water cloud property retrievals. PDFs and scatter plots of $\widehat{R}_{0.86, \mathrm{aA}}, \widehat{R}_{2.1, \mathrm{aA}}$, $\tau_{\mathrm{aA}}$, and $r_{\mathrm{eff}, \mathrm{aA}}$ for both overcast and partially cloudy pixels agree well with their MODIS counterparts, with similar value ranges and mean values. Highly positive correlations between sampled reflectances in the VNIR and SWIR, as well as between $\tau_{\mathrm{M}}$ and $\tau_{\mathrm{aA}}$, are observed with Pearson's product-moment correlation coefficients $R>0.980$. Correlations between $r_{\mathrm{eff}, \mathrm{M}}$ and $r_{\mathrm{eff}, \mathrm{aA}}$ are lower with $R=0.851$, primarily caused by larger differences for partially cloudy pixels. These deviations seem to be retrieval noise caused by increased retrieval uncertainties due to the shape of the LUT for small $\tau_{\mathrm{M}}$ and $\tau_{\mathrm{aA}}$. Limiting the data to only overcast pixels yields $R=0.972$ for the effective droplet radius comparison. However, slight slopes in the reflectance relations yield similar slopes in the cloud product relations, indicating an overestimation of the ASTER results for small values and an underestimation of the ASTER results for larger values. The overall good agreement between the MODIS and ASTER retrievals is confirmed for two example cases. While the rather homogeneous case $\mathrm{C} 14$ is characterized by deviations of \pm 1.82 and $\pm 1.09 \mu \mathrm{m}$ in retrieved cloud optical thickness and effective droplet radius, respectively, partially cloudy pixels sampled for the inhomogeneous case C19 are characterized by differences between $r_{\text {eff,aA }}$ and $r_{\text {eff, }}$ of up to $\pm 12 \mu \mathrm{m}$. Several uncertainty factors in the ASTER and MODIS cloud property comparison are presented.

Not discussed in this study are the comparison of cloud phase and cloud top height retrievals. Since the focus of this study is on MBL scenes over the ocean and the 48 scenes were selected to contain no overlying cirrus, $99 \%$ of all cloudy pixels are characterized to contain liquid water clouds by both ASTER and MODIS. Frequency distributions and statistics of the cloud top height comparison show a high agreement between both instruments. Mean cloud top heights of 670 and $823 \mathrm{~m}$ are observed from ASTER and MODIS, respectively. However, the MODIS cloud top height retrieval is performed at a horizontal resolution of $5 \mathrm{~km}$, averaging observations from a $5 \times 5$ pixel array with a horizontal resolution of $1 \mathrm{~km}$. This means that only 8037 MODIS pixels are included in the analysis (compared to over 150000 ASTER pixels). Further studies with a more comprehensive data set, consisting of different cloud types in different altitudes and with different thermodynamic phases, is required to make a statement about the reliability of the ASTER cloud phase and cloud top height retrieval.

This paper illustrates that the research-level retrieval algorithm for ASTER observations yields reliable cloud prop- 
erty retrievals comparable to the operational MODIS C6 results. The unique ASTER retrievals will enable a number of interesting future studies. ASTER observations at native resolution can help in determining the subpixel cloud structure of heterogeneous cloud pixels, which result in significant uncertainties in the cloud property retrieval (Marshak et al., 2006). Studies by Platnick et al. (2004) and Zhang et al. (2016) show that information about the subpixel reflectance distribution can be used to explain, and possibly correct for, biases in retrieved cloud optical thickness and effective droplet radius. Similar analysis on the distributions of subpixel $\widehat{R}_{0.86, \mathrm{~A}}, \widehat{R}_{2.1, \mathrm{~A}}, \tau_{\mathrm{A}}$, and $r_{\mathrm{eff}, \mathrm{A}}$ can improve the understanding of MODIS PCL retrievals and their uncertainties. Moreover, the ASTER observations at the native resolutions can be aggregated to ever larger horizontal resolutions. Together with the knowledge about the true subpixel cloud structure, such scale-analysis studies will provide valuable insights into 3-D radiative effects and the impacts of resolved and unresolved variability in cloud remote sensing.

\section{Data availability}

The MODIS data are obtained from NASA's Level 1 and Atmosphere Archive and Distribution System (http://ladsweb. nascom.nasa.gov/, LAADS, 2015). ASTER data are obtained by the EarthExplorer interface, courtesy of the NASA EOSDIS Land Processes Distributed Active Archive Center (LP DAAC), USGS/Earth Resources Observation and Science (EROS) Center, Sioux Falls, South Dakota, http: //earthexplorer.usgs.gov (USGS, 2015).

Acknowledgements. This study is supported by NASA grants NNX14AJ25G and NNX15AC77G. The hardware used in the computational studies is part of the UMBC High Performance Computing Facility (HPCF). The facility is supported by the U.S. National Science Foundation through the MRI program (grant nos. CNS-0821258 and CNS-1228778) and the SCREMS program (grant no. DMS-0821311), with additional substantial support from the University of Maryland, Baltimore County (UMBC).

Edited by: A. Sayer

Reviewed by: three anonymous referees

\section{References}

Abrams, M.: The Advanced Spaceborne Thermal Emission and Reflection Radiometer (ASTER): data products for the high spatial resolution imager on NASA's terra platform, Int. J. Remote Sens., 21, 847-859, 2000.

Abrams, M., Hook, S., and Ramachandran, B.: ASTER User Handbook Version 2, Jet Propulsion Laboratory, California Institute of Technology, 4800 Oak Grove Dr., Pasadena, CA 91109, 2004.

Ackerman, S. A., Strabala, K. I., Menzel, W. P., Frey, R. A., Moeller, C. C., and Gumley, L. E.: Discriminating clear sky from clouds with MODIS, J. Geophys. Res., 103, 32141-32157, 1998.
Ackerman, S. A., Holz, R. E., Frey, R., Eloranta, E. W., Maddux, B. C., and McGill, M.: Cloud Detection with MODIS. Part II: Validation, J. Atmos. Ocean. Tech., 25, 1073-1086, doi:10.1175/2007JTECHA1053.1, 2008.

Ardanuy, P. A., Han, D., and Salomonson, V. V.: The Moderate Resolution Imaging Spectrometer (MODIS), IEEE T. Geosci. Remote, 30, 2-27, 1992.

Banks, A. C. and Mélin, F.: An assessment of cloud masking schemes for satellite ocean colour data of marine optical extremes, Int. J. Remote Sens., 36, 797-821, 2015.

Barker, H. and Liu, D.: Inferring optical depth of broken clouds from Landsat data, J. Climate, 8, 2620-2630, 1995.

Barnes, W. L., Pagano, T. S., and Salomonson, V. V.: Prelaunch characteristics of the 'Moderate Resolution Imaging Spectroradiometer' (MODIS) on EOS-AM1, IEEE T. Geosci. Remote, 36, 1088-1100, 1998.

Baum, B. A., Kratz, D. P., Yang, P., Ou, S. C., Hu, Y. X., Soulen, P. F., and Tsay, S. C.: Remote sensing of cloud properties using MODIS airborne simulator imagery during SUCCESS 1. Data and models, J. Geophys. Res., 105, 11767-11780, 2000.

Baum, B. A., Menzel, W. P., Frey, R. A., Tobin, D., Holz, R. E., Ackerman, S. A., Heidinger, A. K., and Yang, P.: MODIS CloudTop Property Refinements for Collection 6, J. Appl. Meteor. Climatol., 51, 1145-1163, doi:10.1175/JAMC-D-11-0203.1, 2012.

Berk, A., Bernstein, L. S., Anderson, G. P., Acharya, P. K., Robertson, D. C., Chetwynd, J. H., and Adler-Golden, S. M.: MODTRAN cloud and multiple scattering upgrades with application to AVIRIS, Remote Sens. Environ., 65, 367-375, 1998.

Cahalan, R., Ridgway, W., Wiscombe, W., and Bell, T.: The albedo of fractal stratocumulus clouds, J. Atmos. Sci., 51, 2434-2455, 1994a.

Cahalan, R., Ridgway, W., Wiscombe, W., Gollmer, S., and Harshvardhan: Independent pixel and Monte Carlo estimates of stratocumulus albedo, J. Atmos. Sci., 51, 3776-3790, 1994b.

Chambers, L., Wielicki, B., and Evans, K.: Accuracy of the independent pixel approximation for satellite estimates of oceanic boundary layer cloud optical depth, J. Geophys. Res., 102, 1779 1794, 1997.

Chevallier, F.: Sampled databases of 60-level atmospheric profiles from the ECMWF analyses, NWPSAF Report, 2002.

Cox, C. and Munk, W.: Measurement of the roughness of the sea surface from photographs of the sun's glitter, J. Opt. Soc. Am. A., 44, 838-850, 1954a.

Cox, C. and Munk, W.: Statistics of the sea surface derived from sun glitter, J. Mar. Res., 13, 198-227, 1954b.

Derber, J. C., Parrish, D. F., , and Lord, S. J.: The New Global Operational Analysis System at the National Meteorological Center, Weather Forecast., 6, 538-547, doi:10.1175/15200434(1991)006<0538:TNGOAS>2.0.CO;2, 1991.

Dey, L., DiGirolamo, L., and Zhao, G.: Scale effect on statistics of the macrophysical properties of trade wind cumuli over the tropical western Atlantic during RICO, J. Geophys. Res., 113, D24214, doi:10.1029/2008JD010295, 2008.

DiGirolamo, L. and Davies, R.: Cloud fraction errors caused by finite resolution measurements, J. Geophys. Res., 102, 1739-1756, 1997.

Frey, R. A., Ackerman, S. A., Liu, Y. H., Strabala, K. I., Zhang, H., Key, J. R., and Wang, X. G.: Cloud detection with MODIS. Part 
I: Improvements in the MODIS cloud mask for collection 5, J. Atmos. Oceanic Technol., 25, 1057-1072, 2008.

Genkova, I., Seiz, G., Zuidema, P., Zhao, G., and DiGirolamo, L.: Cloud top height comparisons from ASTER, MISR, and MODIS for trade wind cumuli, Remote Sens. Environ., 107, 211-222, doi:10.1016/j.rse.2006.07.021, 2007.

Hamann, U., Walther, A., Baum, B., Bennartz, R., Bugliaro, L., Derrien, M., Francis, P. N., Heidinger, A., Joro, S., Kniffka, A., Le Gléau, H., Lockhoff, M., Lutz, H.-J., Meirink, J. F., Minnis, P., Palikonda, R., Roebeling, R., Thoss, A., Platnick, S., Watts, P., and Wind, G.: Remote sensing of cloud top pressure/height from SEVIRI: analysis of ten current retrieval algorithms, Atmos. Meas. Tech., 7, 2839-2867, doi:10.5194/amt-7-2839-2014, 2014.

Heidinger, A. K., Foster, M. J., Walther, A., and Zhao, X.: The Pathfinder-Atmospheres Extended AVHRR climate dataset, B. Am. Meteorol. Soc., 95, 909-922, doi:10.1175/BAMS-D-12$00246.1,2014$.

Huang, C., Townshend, J. R., Liang, S., Kalluri, S. N. V., and DeFries, R. S.: Impact of sensor's point spread function on land cover characterization: assessment and deconvolution, Remote Sens. Environ., 80, 203-212, 2002.

Hulley, G. C. and Hook, S. J.: A new methodology for cloud detection and classification with ASTER data, Geophys. Res. Lett., 35, L16812, doi:10.1029/2008GL034644, 2008.

Hunke, E. C. and Dukowicz, J. K.: An Elastic-Viscous-Plastic Model for Sea Ice Dynamics, J. Phys. Oceanogr., 27, 1849-1867, doi:10.1175/1520-0485(1997)027<1849:AEVPMF>2.0.CO;2, 1997.

Jones, A. L., DiGirolamo, L., and Zhao, G.: Reducing the resolution bias in cloud fraction from satellite derived clearconservative cloud masks, J. Geophys. Res., 117, D12201, doi:10.1029/2011JD017195, 2012.

King, M., Tsay, S.-C., Platnick, S., Wang, M., and Liou, K.-N.: Cloud retrieval algorithms for MODIS: Optical thickness, effective particle radius, and thermodynamic phase, MODIS Algorithm Theoretical Basis Document, No. ATBD-MOD-05, 1997, 1997.

AADS: Level-1 and Atmosphere Archive \& Distribution System/Distributed Active Archive Center, MODIS data, available at: http://ladsweb.nascom.nasa.gov/, 2015.

Loveland, T., Reed, B., Brown, J., Ohlen, D., Zhu, Z., Yang, L., and Merchant, J.: Development of a global land cover characteristics database and IGBP DISCover from $1 \mathrm{~km}$ AVHRR data, Int. J. Remote Sens., 21, 1303-1330, doi:10.1080/014311600210191, 2000.

Marshak, A., Platnick, S., Várnai, T., Wen, G., and Cahalan, B.: Impact of three-dimensional radiative effects on satellite retrievals of cloud droplet sizes, J. Geophys. Res., 111, D09207, doi:10.1029/2005JD006686, 2006.

Mayer, B.: Radiative transfer in the cloudy atmosphere, Eur. Phys. J. Conferences, 1, 75-99, 2009.

Mayer, B. and Kylling, A.: Technical note: The libRadtran software package for radiative transfer calculations -description and examples of use, Atmos. Chem. Phys., 5, 1855-1877, doi:10.5194/acp-5-1855-2005, 2005.

MODIS Characterization Support Team: MODIS LUT Information Guide For Level-1B Version 6.1.14 (TERRA) and Version 6.1.17 (Aqua), NASA/Goddard Space FLight Center, 2012.
Moody, E. G., King, M. D., Platnick, S., Schaaf, C. B., and Gao, F.: Spatially complete global spectral surface albedos: Valueadded datasets derived from terra MODIS land products, IEEE T. Geosci. Remote, 43, 144-158, doi:10.1109/TGRS.2004.838359, 2005.

Moody, E. G., King, M. D., Schaaf, C. B., Hall, D. K., and Platnick, S.: Northern Hemisphere five-year average (2000-2004) spectral albedos of surfaces in the presence of snow: Statistics computed from Terra MODIS land products, Remote Sens. Environ., 111, 337-345, doi:10.1016/j.rse.2007.03.026, 2007.

Moody, E. G., King, M. D., Schaaf, C. B., and Platnick, S.: MODIS-Derived Spatially Complete Surface Albedo Products: Spatial and Temporal Pixel Distribution and Zonal Averages, J. Appl. Meteorol., 47, 2879-2894, doi:10.1175/2008JAMC1795.1, 2008.

Nakajima, T. and King, M.: Determination of the optical thickness and effective particle radius of clouds from reflected solar radiation measurements. Part I: Theory, J. Atmos. Sci., 47, 18781893, 1990.

NASA, (Ed.): US Standard Atmosphere, 1976, US Government Printing Office, Washington, DC, 1976.

Neckel, H. and Labs, D.: Improved data for solar spectral irradiance from 0.33 to $1.25 \mu$, Sol. Phys., 74, 231-249, 1984.

Nolin, A., Armstrong, R. L., and Maslanik, J.: Near-Real-Time SSM/I-SSMIS EASE-Grid Daily Global Ice Concentration and Snow Extent, Version 4, [Extent], Boulder, Colorado USA, NASA DAAC at the National Snow and Ice Data Center, 1998.

Platnick, S.: Vertical photon transport in cloud remote sensing problems, J. Geophys. Res., 105, 22919-22935, 2000.

Platnick, S., King, M., Ackerman, S., Menzel, W., Baum, B., Riedi, J., and Frey, R.: The MODIS cloud products: Algorithms and examples from TERRA, IEEE Trans. Geosci. Remote Sens., 41, 459-473, 2003.

Platnick, S., Pincus, R., Wind, B., King, M. D., Gray, M., and Hubanks, P.: An initial analysis of the pixel-level uncertainties in global MODIS cloud optical thickness and effective particle size retrievals, Proc. SPIE, 5652, 30-40, doi:10.1117/12.578353, 2004.

Rossow, W. and Schiffer, R.: ISCCP cloud data products, B. Am. Meteorol. Soc., 72, 2-20, 1991

Saunders, R. and Kriebel, K.: An improved method for detecting clear sky and cloudy radiances from AVHRR data, Int. J. Remote Sens., 9, 123-150, 1988.

Seemann, S. W., Borbas, E. E., Knuteson, R. O., Stephenson, G. R., and Huang, H. L.: Development of a global infrared land surface emissivity database for application to clear sky sounding retrievals from multispectral satellite radiance measurements, J. App. Meteor. Climatol., 47, 108-123, doi:10.1175/2007JAMC1590.1, 2008.

Seiz, G., Davies, R., and Grün, A.: Stereo cloud-top height retrieval with ASTER and MISR, Int. J. Remote Sens., 27, 1839-1853, doi:10.1080/01431160500380703, 2006.

Shenk, W. and Salomonson, V.: A simulation study exploring the effects of sensor spatial resolution on estimates of cloud cover from satellite, J. Appl. Meteorol., 11, 214-220, 1972.

Stamnes, K., Tsay, S., Wiscombe, W., and Jayaweera, K.: A numerically stable algorithm for discrete-ordinate-method radiative transfer in multiple scattering and emitting layered media, Appl. Opt., 27, 2502-2509, 1988. 
Stamnes, K., Tsay, S.-C., Wiscombe, W., and Laszlo, I.: DISORT, a General-Purpose Fortran Program for Discrete-Ordinate-Method Radiative Transfer in Scattering and Emitting Layered Media: Documentation of Methodology, Tech. rep., Dept. of Physics and Engineering Physics, Stevens Institute of Technology, Hoboken, NJ 07030, 2000.

Stefanov, W. L. and Netzband, M.: Assessment of ASTER land cover and MODIS NDVI data at multiple scales for ecological characterization of an arid urban center, Remote Sens. Environ., 99, 31-43, 2005.

Strow, L., Hannon, S., Machado, S., Motteler, H., and Tobin, D.: An overview of the AIRS radiative transfer model, IEEE T. Geosci. Remote Sens., 41, 303-313, doi:10.1109/TGRS.2002.808244, 2003.

Sun, J., Xiong, X., Che, N., and Angal, A.: Terra MODIS Band 2 Electronic Crosstalk: Cause, Impact, and Mitigation, Proc. SPIE, 7826, 78261Y-1-78261Y-10, 2010.

Sun, J., Angal, A., Xiong, X., Chen, H., Geng, X., Wu, A., Choi, T., and Chu, M.: MODIS Reflective Solar Bands Calibration Improvements in Collection 6, Proc. SPIE, 8528, 85280N, doi:10.1117/12.979733, 2012.

Sun, J., Xiong, X., Madhavan, S., and Wenny, B. N.: Terra MODIS Band 27 Electronic Crosstalk Effect and Its Removal, IEEE T. Geosci. Remote Sens., 52, 1551-1561, 2014.

Thekaekara, M.: Extraterrestrial solar spectrum, 3000-6100A at 1$\AA$ Antervals, Appl. Opt., 13, 518-522, 1974.

Thuillier, G., Hersé, M., Labs, D., Foujols, T., Peetermans, W., Gillotay, D., Simon, P. C., and Mandel, H.: The solar spectral irradiance from 200 to $2400 \mathrm{~nm}$ as measured by the solspec spectrometer from the ATLAS and EURECA missions, Sol. Phys., 214, 1-22, 2003.

Twomey, S. and Seton, K. J.: Inferences of gross microphysical properties of clouds from spectral reflectance measurements, J. Atmos. Sci., 37, 1065-1069, 1980.

SGS (Earth Resources Observation and Science): ASTER data, available at: http://earthexplorer.usgs.gov, 2015.

Uprety, S., Cao, C., Xiong, X., Blonski, S., Wu, A., and Shao, X.: Radiometric Intercomparison between Suomi-NPP VIIRS and Aqua MODIS Reflective Solar Bands Using Simultaneous Nadir Overpass in the Low Latitudes, J. Atmos. Ocean. Technol., 30, 2720-2736, 2013.

Wang, M. and King, M.: Correction of Rayleigh scattering effects in cloud optical thickness retrievals, J. Geophys. Res., 102, 2591525926, doi:10.1029/97JD02225, 1997.

Wen, G., Marshak, A., Cahalan, R. F., Remer, L. A., and Kleidman, R. G.: 3-D aerosol-cloud radiative interaction observed in collocated MODIS and ASTER images of cumulus cloud fields, J. Geophys. Res., 112, D13204, doi:10.1029/2006JD008267, 2007.

Werner, F., Ditas, F., Siebert, H., Simmel, M., Wehner, B., Pilewskie, P., Schmeissner, T., Shaw, R. A., Hartmann, S., Wex, H., Roberts, G. C., and Wendisch, M.: Twomey effect observed from collocated microphysical and remote sensing measurements over shallow cumulus, J. Geophys. Res., 119, 1534-1545, doi:10.1002/2013JD020131, 2014.
Wielicki, B. and Welch, R.: Cumulus cloud properties derived using Landsat satellite data, J. Climate Appl. Meteor., 25, 261-276, 1986.

Wielicki, B. A. and Parker, L.: On the determination of cloud cover from satellite sensors: The effect of sensor spatial resolution, J. Geophys. Res., 97, 12799-12823, 1992.

Wind, G., Platnick, S., King, M., Hubanks, P., Pavolonis, M., Heidinger, A., Baum, B., and Yang, P.: Multilayer Cloud Detection with the MODIS Near-Infrared Water Vapor Absorption Band, J. Appl. Meteor. Climatol., 49, 2315-2333, doi:10.1175/2010JAMC2364.1, 2010.

Wiscombe, W.: Improved Mie scattering algorithms, Appl. Opt., 19, 1505-1509, 1980.

Xiong, X., Sun, J., Chiang, K., Xiong, S., and Barnes, W.: MODIS on-orbit characterization using the moon, Sensors, Systems And Next-Generation Satellites Vi, 4881, 299-307, 2003.

Xiong, X., Wenny, B. N., and Barnes, W. L.: Overview of NASA Earth Observing Systems Terra and Aqua moderate resolution imaging spectroradiometer instrument calibration algorithms and on-orbit performance, J. Appl. Remote Sens., 3, 032501 , doi:10.1117/1.3180864, 2009.

Yamaguchi, Y. and Hiroji, T.: Scientific basis of ASTER instrument design, Proc. SPIE, 1939, 150-160, 1993.

Yamaguchi, Y., Kahle, A., Tsu, H., Kawakami, T., and Pniel, M. Overview of Advanced Spaceborne Thermal Emission and Reflection Radiometer (ASTER), IEEE Trans. Geosci. Remote Sens., 36, 1062-1071, 1998.

Yang, Y. and Di Girolamo, L.: Impacts of 3-D radiative effects on satellite cloud detection and their consequences on cloud fraction and aerosol optical depth retrievals, J. Geophys. Res., 113, 032501-1-032501-25, 2008

Zhang, Z. and Platnick, S.: An assessment of differences between cloud effective particle radius retrievals for marine water clouds from three MODIS spectral bands, J. Geophys Res., 116, D04213, doi:10.1029/2007JD009095, 2011.

Zhang, Z., Ackerman, A. S., Feingold, G., Platnick, S., Pincus, R., and Xue, H.: Effects of cloud horizontal inhomogeneity and drizzle on remote sensing of cloud droplet effective radius: Case studies based on large-eddy simulations, J. Geophys. Res., 117, D19208, doi:10.1029/2012JD017655, 2012.

Zhang, Z., Werner, F., Cho, H.-M., Wind, G., Platnick, S., Ackerman, A. S., Di Girolamo, L., Marshak, A., and Meyer, K.: A framework based on 2-D Taylor expansion for quantifying the impacts of subpixel reflectance variance and covariance on cloud optical thickness and effective radius retrievals based on the bispectral method, J. Geophys. Res. Atmos., 121, 7007-7025, doi:10.1002/2016JD024837, 2016.

Zhao, G. and Di Girolamo, L.: Cloud fraction errors for trade wind cumuli from EOS-Terra instruments, Geophys. Res. Lett., 33, L20802, doi:10.1029/2006GL027088, 2006.

Zhao, G. and Di Girolamo, L.: Statistics on the macrophysical properties of trade wind cumuli over the tropical western Atlantic, J. Geophys. Res., 112, D10204, doi:10.1029/2006JD007371, 2007. 